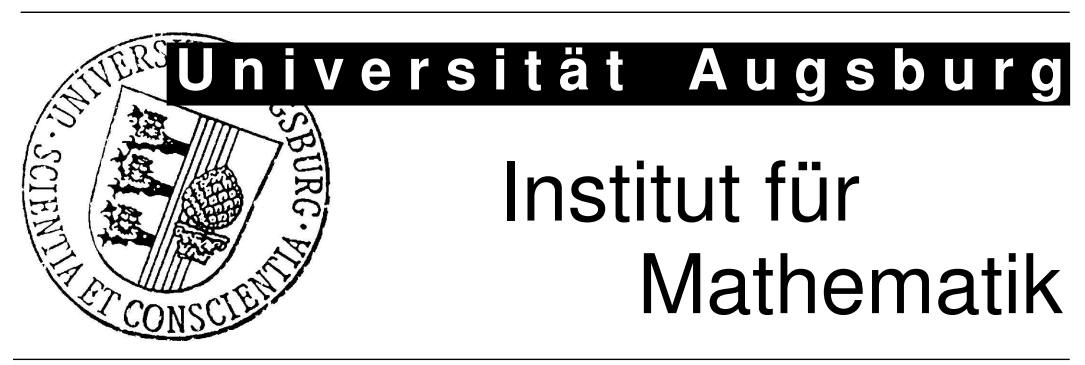

Lars Diening, Christian Kreuzer

Linear convergence of an adaptive finite element method for the $\boldsymbol{p}$-Laplacian equation 


\section{Impressum:}

Herausgeber:

Institut für Mathematik

Universität Augsburg

86135 Augsburg

http://www . math. uni-augsburg.de/forschung/preprint/

ViSdP:

Christian Kreuzer

Institut für Mathematik

Universität Augsburg

86135 Augsburg

Preprint: Sämtliche Rechte verbleiben den Autoren (C) 2007 


\title{
LINEAR CONVERGENCE OF AN ADAPTIVE FINITE ELEMENT METHOD FOR THE $p$-LAPLACIAN EQUATION
}

\author{
LARS DIENING AND CHRISTIAN KREUZER
}

\begin{abstract}
We study an adaptive finite element method for the $p$-Laplacian like PDE's using piecewise linear, continuous functions. The error is measured by means of the quasi-norm of Barrett and Liu. We provide residual based error estimators without a gap between the upper and lower bound. We show linear convergence of the algorithm which is similar to the one of Morin, Nochetto and Siebert. All results are obtained without extra marking for the oscillation.
\end{abstract}

\section{INTRODUCTION}

Let $\Omega$ be a polyhedral, bounded domain in $\mathbb{R}^{d}, d \in \mathbb{N}$. We consider the following system of nonlinear structure

$$
\begin{aligned}
-\operatorname{div}(\mathbf{A}(\nabla u)) & =f & & \text { in } \Omega, \\
u & =0 & & \text { on } \partial \Omega .
\end{aligned}
$$

Our considerations include in particular the case of the $p$-Laplacian, where

$$
\mathbf{A}(\nabla u)=(\kappa+|\nabla u|)^{p-2} \nabla u,
$$

with $1<p<\infty, \kappa \geq 0, f \in L^{p^{\prime}}(\Omega)$, and $\frac{1}{p}+\frac{1}{p^{\prime}}=1$.

The purpose of this paper is to present a linear convergence result for an adaptive finite element method AFEM applied to the nonlinear Laplace equation (1.1). As is common practice the adaptive finite element method consists of a loop

$$
\mathrm{SOLVE} \rightarrow \text { EstimaTE } \rightarrow \text { MARK } \rightarrow \text { ReFine }
$$

starting from a initial triangulation of $\Omega$. To be more specific, the finite element problem on the current mesh is solved, then the aposteriori error estimator is computed and finally with its help elements are marked for refinement. The marking strategy resorts to Dörfler's marking introduced in [Dör96]. The algorithm uses piecewise linear, continuous finite elements, whereas the refinement is realized by newest vertex bisection. This produces a sequence of weak finite element solutions $u_{k}$ of (1.1) in nested finite element spaces $V_{k}$.

The main result states linear convergence of $u_{k}$ to the weak solution $u$ of (1.1). In particular, we show that there exists $\alpha \in(0,1), C>0$ with

$$
\left\|\mathbf{F}\left(\nabla u_{k}\right)-\mathbf{F}(\nabla u)\right\|_{2}^{2}+\operatorname{osc}_{k}^{2}(f) \leq \alpha^{2 k} C
$$

where the vector field $\mathbf{F}$ arises from the vector field $\mathbf{A}$ by $\mathbf{F}(\mathbf{a}):=|\mathbf{A}(\mathbf{a})|^{\frac{1}{2}}|\mathbf{a}|^{-\frac{1}{2}} \mathbf{a}$. The $L^{2}$ norm of the error $\left\|\mathbf{F}\left(\nabla u_{k}\right)-\mathbf{F}(\nabla u)\right\|_{2}^{2}$ measured in terms of $\mathbf{F}$ is equivalent to the so called quasi norm $\left\|\nabla u_{k}-\nabla u\right\|_{(p)}^{2}$ introduced by Barrett and Liu,

Date: July 4, 2007.

2000 Mathematics Subject Classification. 65N15, 65N30, 35J60, 35J70.

Key words and phrases. Convergence, a posteriori error estimator, p-Laplacian, quasi norms, error reduction, oscillation.

L. Diening is indebted to the Landesstiftung Baden-Württemberg for facilitating the analysis entailed in this paper.

C. Kreuzer has been supported by Project C.1 of the DFG-Research-Unit "Nonlinear Partial Differential Equations" Generalized Newtonian fluids and electrorheological fluids. 
cf. [BL94a] and Remark 4. The quasi norm was a breakthrough in the numerical investigation of (1.1). In particular, Barrett and Liu obtained the best approximation property of the conforming, finite element solution $u_{h} \in V_{h}$ in terms of quasi norms, i.e.

$$
\left\|\nabla v-\nabla v_{h}\right\|_{(p)}^{2} \leq c \min _{\psi_{h} \in V_{h}}\left\|\nabla v-\nabla \psi_{h}\right\|_{(p)}^{2},
$$

In [EL05] it has been proved by Ebmeyer and Liu that for piecewise linear, continuous finite elements and $p>\frac{2 d}{d+2}$ the best approximation error can be estimated as

$$
\min _{\psi_{h} \in V_{h}}\left\|\nabla v-\nabla \psi_{h}\right\|_{(p)}^{2} \leq c h^{2} \int_{\Omega}(\kappa+|\nabla u|)^{p-2}\left|\nabla^{2} u\right|^{2} d x .
$$

Recently, Diening and Růžička improved these results in [DR06] to the case $p>1$ admitting also more general finite element spaces. In particular, they showed

$$
\left\|\mathbf{F}(\nabla v)-\mathbf{F}\left(\nabla v_{h}\right)\right\|_{2}^{2} \leq c \min _{\psi_{h} \in V_{h}}\left\|\mathbf{F}(\nabla v)-\mathbf{F}\left(\nabla \psi_{h}\right)\right\|_{2}^{2}
$$

and

$$
\left\|\mathbf{F}(\nabla v)-\mathbf{F}\left(\nabla \Pi_{h} v\right)\right\|_{2}^{2} \leq c h^{2}\|\nabla \mathbf{F}(\nabla u)\|_{2}^{2},
$$

where $\Pi_{h}$ is a suitable interpolation operator, e.g. the Scott-Zhang operator. We want to mention that the right hand sides of (1.3) and (1.4) are proportional. They express the natural regularity of a strong solution of (1.1) (cf. [Giu03], [BL93a], [ELS05], [Ebm05]).

The technique of quasi-norms founds its way into a posteriori analysis in the work of Liu and Yan [LY01, LY02]. They show that

$$
c \eta_{h}^{2}-C \operatorname{osc}_{h}^{2}(f) \leq\left\|\nabla u-\nabla u_{h}\right\|_{(p)}^{2} \leq C\left(\eta_{h}^{2}+\widetilde{\eta}^{2}\right)
$$

for residual based estimators $\eta_{h}$. Numerical experiments [LY01, CK06] indicate, that these new estimators are indeed sharper and lead to more efficient meshes than existing ones. However for convergence analysis the additional term $\widetilde{\eta}^{2}$ causes problems, since it forms a gap between the left and the right hand side. In this work we are able to overcome this drawback and prove estimates avoiding $\widetilde{\eta}^{2}$ (see Lemma 8 and Corollary 11):

$$
c \eta_{h}^{2}-C \operatorname{osc}_{h}^{2}(f) \leq\left\|\mathbf{F}(\nabla u)-\mathbf{F}\left(\nabla u_{h}\right)\right\|_{2}^{2} \leq C \eta_{h}^{2} .
$$

Recently a posteriori error estimators by gradient recovery have been studied in [CLY06].

Dörfler was the first who proved in [Dör96] linear error reduction of (AFEM) for the linear Laplacian, if the data oscillation is small enough. Later, this additional assumption has been removed by Morin, Nochetto, and Siebert in [MNS00] by additional marking for oscillation. The first convergence result for the nonlinear Laplacian is stated by Veeser [Ve02]. There residual based estimators for the $W^{1, p}$ norm are used. Since there appears a gap in the power between the upper and lower estimates this prevents to prove linear convergence.

The convergence results in the linear case are heavily based on Galerkin orthogonality and the Pythagorean Theorem which yield

$$
\left\|u_{h}-u\right\|^{2}=\|\| u_{H}-u\|\|^{2}-\left\|u_{H}-u_{h}\right\|^{2} .
$$

in the energy norm. To overcome the lack of orthogonality in the non-linear case we proceed as follows: We prove that the energy difference of weak solutions in nested spaces $V_{1} \subset V_{2}$ is proportional to the quasi-norm distance, i.e.

$$
\mathcal{J}\left(u_{1}\right)-\mathcal{J}\left(u_{2}\right) \sim\left\|\nabla u_{1}-\nabla u_{2}\right\|_{(p)}^{2} \sim\left\|\mathbf{F}\left(\nabla u_{1}\right)-\mathbf{F}\left(\nabla u_{2}\right)\right\|_{2}^{2},
$$


where $\mathcal{J}(u)$ is the energy functional of (1.1), $u_{1} \in V_{1}$, and $u_{2} \in V_{2}$, see (2.10) and Lemma 16. This property and the trivial equality

$$
\mathcal{J}\left(u_{h}\right)-\mathcal{J}(u)=\left(\mathcal{J}\left(u_{H}\right)-\mathcal{J}(u)\right)-\left(\mathcal{J}\left(u_{H}\right)-\mathcal{J}\left(u_{h}\right)\right)
$$

is our substitute for the orthogonality of the error (1.6).

In the linear, symmetric case it is possible to consider the reduction of the error and the oscillation independently, since the oscillation is solely dependent on the data $f$. Mekchay and Nochetto showed linear reduction of the sum of error and oscillation for non-symmetric second order linear elliptic PDE in [MN05]. In this case oscillation and error are coupled. A similar effect appears in our non-linear setting. We introduce a new proof for error reduction, which enables us to manage without extra marking for oscillation, see Remark 14. Our proof permits to use the fact that oscillation is dominated by the error indicator. Moreover, we prove a strict reduction of the difference of energies plus the oscillation in each step.

An essential tool in our calculations in the use of shifted $\mathrm{N}$-functions, namely $\varphi_{a}$. They are closely related to the quasi-norms, which is best expressed by the relation

$$
(\mathbf{A}(\mathbf{a})-\mathbf{A}(\mathbf{b})) \cdot(\mathbf{a}-\mathbf{b}) \sim|\mathbf{F}(\mathbf{a})-\mathbf{F}(\mathbf{b})|^{2} \sim \varphi_{|\mathbf{a}|}(|\mathbf{a}-\mathbf{b}|)
$$

for $\mathbf{a}, \mathbf{b} \in \mathbb{R}^{d}$. See Lemma 3 for more details. The shifted N-functions enable us to handle more general non-linear equations than the $p$-Laplacian, namely the $\varphi$ Laplacian from (2.1). But most important, the shifted N-functions simplify and clarify the calculations significantly also in the case of the $p$-Laplacian.

\section{Preliminaries}

We first introduce the nonlinear Dirichlet problem. Thereby the nonlinear partial differential operator called $\varphi$-Laplacian is defined via a certain function $\varphi: \mathbb{R} \geq 0 \rightarrow$ $\mathbb{R}^{\geq 0}$. The most popular case of such operators is the $p$-Laplacian which corresponds to the function $\varphi(t):=\int_{0}^{t}(\kappa+s)^{p-2} s d s$. As mentioned before the treatment of the nonlinear Laplacian via $N$-functions simplifies and clarifies calculations. Assumptions on $\varphi$ and related properties are discussed subsequently. Afterwards the weak formulation of the problem is stated along with the corresponding minimizing problem.

Let $\Omega$ be a polyhedral, bounded domain in $\mathbb{R}^{d}, d \in \mathbb{N}$. In the center of our considerations are solutions of the $\varphi$-Laplacian problem, i.e.

$$
\begin{aligned}
-\operatorname{div}(\mathbf{A}(\nabla u)) & =f & & \text { in } \Omega, \\
u & =0 & & \text { on } \partial \Omega,
\end{aligned}
$$

with

$$
\mathbf{A}(\nabla u)=\varphi^{\prime}(\mid \nabla u) \mid \frac{\nabla u}{|\nabla u|} .
$$

2.1. Assumptions on $\varphi$ and resulting properties. Now we shed light on the considered function $\varphi$. To go not beyond the scope of this work we give only a short sketch of the underlying theory. The following definitions and results are standard in the theory of Orlicz functions and can for example be found in [RR91]. A few assertions are also proved at the Appendix 5.

We use $c, C>0$ (no index) as generic constants, i.e. their value may change from line to line but does not depend on the important variables. Furthermore, we write $f \sim g$ iff $c f \leq g \leq C f$.

A continuous function $\varphi: \mathbb{R}^{\geq 0} \rightarrow \mathbb{R}^{\geq 0}$ is said to be an $N$-function, iff

- $\varphi$ is continuous and convex with $\varphi(0)=0$;

- there exists a derivative $\varphi^{\prime}$ of $\varphi$ which is right continuous, non-decreasing and satisfies $\varphi^{\prime}(0)=0, \varphi^{\prime}(t)>0$ for $t>0$, and $\lim _{t \rightarrow \infty} \varphi^{\prime}(t)=\infty$. 
The $\mathrm{N}$ in $\mathrm{N}$-function stands for nice function. Furthermore, $\varphi$ satisfies the $\Delta_{2^{-}}$ condition, iff it holds

- $\varphi(2 t) \leq C \varphi(t)$ uniformly in $t \geq 0$.

We denote the smallest such constant by $\Delta_{2}(\varphi)$. Since $\varphi(t) \leq \varphi(2 t)$ the $\Delta_{2^{-}}$ condition means that $\varphi(t)$ and $\varphi(2 t)$ are proportional. Note that if $\Delta_{2}(\varphi)<\infty$ then $\varphi(t) \sim \varphi(a t)$ uniformly in $t \geq 0$ for any fixed $a>1$.

Define $\left(\varphi^{\prime}\right)^{-1}: \mathbb{R}^{\geq 0} \rightarrow \mathbb{R}^{\geq 0}$ as

$$
\left(\varphi^{\prime}\right)^{-1}(t):=\sup \left\{u \in \mathbb{R}^{\geq 0}: \varphi^{\prime}(u) \leq t\right\} .
$$

If $\varphi^{\prime}$ is strictly increasing then $\left(\varphi^{\prime}\right)^{-1}$ is the inverse function of $\varphi^{\prime}$. By the definition

$$
\varphi^{*}(t):=\int_{0}^{t}\left(\varphi^{\prime}\right)^{-1}(s) d s
$$

the so called complementary function $\varphi^{*}$ of $\varphi$ is again an N-function and $\left(\varphi^{*}\right)^{\prime}(t)=$ $\left(\varphi^{\prime}\right)^{-1}(t)$ for $t>0$. Note that $\left(\varphi^{*}\right)^{*}=\varphi$.

Assume that $\Delta_{2}(\varphi), \Delta_{2}\left(\varphi^{*}\right)<\infty$. Then for all $\delta>0$ there exists $C_{\delta}>0$ (only depending on $\Delta_{2}(\varphi)$, and $\left.\Delta_{2}\left(\varphi^{*}\right)\right)$, such that for all $s, t \geq 0$ hold

$$
\begin{aligned}
& s t \leq C_{\delta} \varphi(s)+\delta \varphi^{*}(t), \\
& s t \leq \delta \varphi(s)+C_{\delta} \varphi^{*}(t) .
\end{aligned}
$$

This inequalities are called Young's inequality. Moreover, for all $t>0$ there exists $s>0$ such that

$$
s t=\varphi(s)+\varphi^{*}(t) .
$$

Further basic inequalities are for all $t \geq 0$

$$
\begin{gathered}
t \leq \varphi^{-1}(t)\left(\varphi^{*}\right)^{-1}(t) \leq 2 t, \\
\frac{t}{2} \varphi^{\prime}\left(\frac{t}{2}\right) \leq \varphi(t) \leq t \varphi^{\prime}(t), \\
\varphi\left(\frac{\varphi^{*}(t)}{t}\right) \leq \varphi^{*}(t) \leq \varphi\left(\frac{2 \varphi^{*}(t)}{t}\right) .
\end{gathered}
$$

Therefor, uniformly in $t \geq 0$

$$
\varphi^{-1}(t)\left(\varphi^{*}\right)^{-1}(t) \sim t, \quad \varphi(t) \sim \varphi^{\prime}(t) t, \quad \varphi^{*}\left(\varphi^{\prime}(t)\right) \sim \varphi(t),
$$

where the constants only depend on $\Delta_{2}(\varphi)$ and $\Delta_{2}\left(\varphi^{*}\right)$.

As in [DE05, DR06] we require the following properties about our function $\varphi$ :

Assumption 1. Let $\varphi$ be an N-function with $\Delta_{2}(\varphi)<\infty, \Delta_{2}\left(\varphi^{*}\right)<\infty$, and $\varphi \in C^{2}(0, \infty)$ such that

$$
\varphi^{\prime}(t) \sim t \varphi^{\prime \prime}(t)
$$

uniformly in $t \geq 0$.

It is shown in [DE05] that $\varphi$ satisfies Assumption 1 if and only if $\varphi^{*}$ satisfies Assumption 1. Moreover, it is shown that we have for all $\mathbf{a}, \mathbf{b} \in \mathbb{R}^{d}$

$$
\begin{aligned}
(\mathbf{A}(\mathbf{a})-\mathbf{A}(\mathbf{b})) \cdot(\mathbf{a}-\mathbf{b}) & \geq c \varphi^{\prime \prime}(|\mathbf{a}|+|\mathbf{b}|)|\mathbf{a}-\mathbf{b}|^{2}, \\
|\mathbf{A}(\mathbf{a})-\mathbf{A}(\mathbf{b})| & \leq C \varphi^{\prime \prime}(|\mathbf{a}|+|\mathbf{b}|)|\mathbf{a}-\mathbf{b}|,
\end{aligned}
$$

where $c, C$ only depend on $\Delta_{2}(\varphi), \Delta_{2}(\varphi)$, and the constant in (2.7).

Remark 2. The most important example of such functions is certainly the pLaplacian. Thereby $\varphi(t):=\int_{0}^{t}(\kappa+s)^{p-2} s d s$ with $1<p<\infty$ and $\kappa \geq 0$. This 
function satisfies Assumption 1. If $\kappa=0$, then Young's inequality (2.3) coincides with the well known classical Young's inequality

$$
s t \leq \delta \frac{1}{p} t^{p}+\delta^{\frac{1}{p-1}} \frac{1}{q} s^{q},
$$

where $q \in(1, \infty)$ with $\frac{1}{p}+\frac{1}{q}=1$. Moreover, with $\mathbf{A}(\mathbf{a})=\varphi^{\prime}(|\mathbf{a}|) \frac{\mathbf{a}}{|\mathbf{a}|}=(\kappa+|\mathbf{a}|)^{p-2} \mathbf{a}$ (2.8) corresponds to the well known monotonicity and coercivity inequalities

$$
\begin{aligned}
\left((\kappa+|\mathbf{a}|)^{p-2} \mathbf{a}-(\kappa+|\mathbf{b}|)^{p-2} \mathbf{b}\right)(\mathbf{a}-\mathbf{b}) & \geq c(\kappa+|\mathbf{a}|+|\mathbf{b}|)^{p-2}|\mathbf{a}-\mathbf{b}|^{2}, \\
\left|(\kappa+|\mathbf{a}|)^{p-2} \mathbf{a}-(\kappa+|\mathbf{b}|)^{p-2} \mathbf{b}\right| & \leq C(\kappa+|\mathbf{a}|+|\mathbf{b}|)^{p-2}|\mathbf{a}-\mathbf{b}| .
\end{aligned}
$$

for all $\mathbf{a}, \mathbf{b} \in \mathbb{R}^{d}$ (see e.g. [GM75, BL93a]).

2.2. Weak formulation of the $\varphi$-Laplacian problem and corresponding minimizing problem. We introduce the weak form of the nonlinear equation (2.1). To proceed so we first have to introduce analytical background. For details we refer to [RR91].

In the following the function $\varphi$ will be a fixed N-function as stated in Assumption 1. By $L^{\varphi}$ and $W^{1, \varphi}$ we denote the classical Orlicz and Sobolev-Orlicz spaces i.e. $g \in L^{\varphi}$ iff $\int \varphi(|g|) d x<\infty$ and $g \in W^{1, \varphi}$ iff $g, \nabla g \in L^{\varphi}$. The norm on $L^{\varphi}$ is given by $\|f\|_{\varphi}=\inf \left\{\lambda>0: \int \varphi(f / \lambda) d x \leq 1\right\}$. By $W_{0}^{1, \varphi}(\Omega)$ we denote the closure of $C_{0}^{\infty}(\Omega)$ in $W^{1, \varphi}(\Omega)$.

The weak formulation reads as follows: For $f \in L^{\varphi^{*}}(\Omega) \subset\left(W_{0}^{1, \varphi}(\Omega)\right)^{*}$ find $u \in W_{0}^{1, \varphi}(\Omega)$ with

$$
\langle\mathbf{A}(\nabla u), \nabla v\rangle=\langle f, v\rangle \quad \text { for all } v \in W_{0}^{1, \varphi}(\Omega) .
$$

The theory of monotone operators ensures the unique existence of $u$. Moreover, $u$ is the unique minimizer of the energy functional

$$
\mathcal{J}(u):=\int_{\Omega} \varphi(|\nabla u|) d x-\int_{\Omega} u f d x \rightarrow \min !
$$

\section{A Posteriori Analysis}

In this section we first dicuss our concept of distance. In particular we introduce shifted $\mathrm{N}$-functions and construct an measure of distance related to the nonlinear problem (2.1). In case of the $p$-Laplacian this concept is equivalent to the quasinorm introduced by Barrett and Liu [BL93a]. In the following we introduce the finite element spaces and note an interpolation inequality. In the second part of this section we construct a posteriori upper and lower bounds for the error of a finite element solution to the continuous solution. Finally, we discuss a discrete local lower bound, i.e., a lower bound for the distance between two different finite element solutions.

3.1. Concept of Distance. Let $\varphi$ be again a fixed N-function. We define another $\mathrm{N}$-function $\psi$ by

$$
\psi^{\prime}(t):=\sqrt{\varphi^{\prime}(t) t}
$$

and set for $\mathbf{a} \in \mathbb{R}^{d}$

$$
\mathbf{F}(\mathbf{a}):=\psi^{\prime}(|\mathbf{a}|) \frac{\mathbf{a}}{|\mathbf{a}|}=\sqrt{\varphi^{\prime}(|\mathbf{a}|)|\mathbf{a}|} \frac{\mathbf{a}}{|\mathbf{a}|}=\sqrt{|\mathbf{A}(\mathbf{a})||\mathbf{a}|} \frac{\mathbf{a}}{|\mathbf{a}|} .
$$

It is shown in [DE05] that since $\varphi$ satisfies Assumption 1 also $\psi, \psi^{*}$, and $\varphi^{*}$ satisfy Assumption 1. It is also shown that as a consequence (2.8) holds with $\mathbf{A}, \varphi$ replaced by $\mathbf{F}, \psi$. In addition we introduce a family of N-function $\left\{\varphi_{a}\right\}_{a \geq 0}$ by

$$
\frac{\varphi_{a}^{\prime}(t)}{t}:=\frac{\varphi^{\prime}(a+t)}{a+t}
$$


which owing to (2.7) implies $\varphi_{a}^{\prime \prime}(t) \sim \varphi^{\prime \prime}(a+t)$ uniformly in $a, t \geq 0$. The functions $\varphi_{a}$ are called shifted $\mathrm{N}$-functions. The basic properties of $\varphi_{a}$ are summarized in the appendix. The connection between $\mathbf{A}, \mathbf{F}$, and $\left\{\varphi_{a}\right\}_{a \geq 0}$ is best reflected in the following lemma from [DE05].

Lemma 3. Let $\varphi$ satisfy Assumption 1 and let $\mathbf{A}$ and $\mathbf{F}$ be defined by (2.2) and (3.1). Then

$$
\begin{aligned}
(\mathbf{A}(\mathbf{a})-\mathbf{A}(\mathbf{b})) \cdot(\mathbf{a}-\mathbf{b}) & \sim|\mathbf{F}(\mathbf{a})-\mathbf{F}(\mathbf{b})|^{2} \\
& \sim \varphi_{|\mathbf{a}|}(|\mathbf{a}-\mathbf{b}|), \\
& \sim|\mathbf{a}-\mathbf{b}|^{2} \varphi^{\prime \prime}(|\mathbf{a}|+|\mathbf{b}|),
\end{aligned}
$$

uniformly in $\mathbf{a}, \mathbf{b} \in \mathbb{R}^{d}$. If $\varphi^{\prime \prime}(0)$ does not exists, the expression in (3.3c) is continuously extended by zero for $|\mathbf{a}|=|\mathbf{b}|=\mathbf{0}$. Moreover

$$
\begin{aligned}
|\mathbf{A}(\mathbf{a})-\mathbf{A}(\mathbf{b})| & \sim \varphi_{|\mathbf{a}|}^{\prime}(|\mathbf{a}-\mathbf{b}|), \\
\mathbf{A}(\mathbf{b}) \cdot \mathbf{b} \sim|\mathbf{F}(\mathbf{b})|^{2} & \sim \varphi(|\mathbf{b}|)
\end{aligned}
$$

uniformly in $\mathbf{a}, \mathbf{b} \in \mathbb{R}^{d}$.

We use the equivalences (3.3) extensively in this paper without referring.

An immediately consequence of Lemma 3 is

Corollary 4. We have for all $u, v \in W^{1, \varphi}(\Omega)$

$$
\begin{aligned}
\int_{\Omega}(\mathbf{A}(\nabla u)-\mathbf{A}(\nabla v)) \cdot(\nabla u-\nabla v) d x & \sim\|\mathbf{F}(\nabla u)-\mathbf{F}(\nabla v)\|_{2}^{2} \\
& \sim \int_{\Omega} \varphi|\nabla u|(|\nabla u-\nabla v|) d x .
\end{aligned}
$$

Remark 5. In the case of the p-Laplacian, i.e. $\mathbf{A}(\nabla v)=(\kappa+|\nabla v|)^{p-2} \nabla v$ and $\varphi^{\prime}(t)=(\kappa+t)^{p-2} t$ with $1<p<\infty$ and $\kappa \geq 0$ we have for all $\mathbf{a} \in \mathbb{R}^{d}, t \geq 0$

$$
\mathbf{F}(\mathbf{a}):=(\kappa+|\mathbf{a}|)^{\frac{p-2}{2}} \mathbf{a}, \quad \quad \psi^{\prime}(t):=(\kappa+t)^{\frac{p-2}{2}} t .
$$

Moreover, for the p-Laplacian all expressions in Corrolary 4 are proportional to the quasi-norm introduced by Barrett and Liu in [BL93a]. This follows from the relation

$$
\varphi_{|\mathbf{a}|}^{\prime}(t)=(\kappa+|\mathbf{a}|+t)^{p-2} t
$$

and

$$
\begin{aligned}
\|\nabla u-\nabla v\|_{(p)}^{2} & =\int_{\Omega}(\kappa+|\nabla u|+|\nabla u-\nabla v|)^{p-2}|\nabla u-\nabla v|^{2} d x \\
& =\int_{\Omega} \varphi_{|\nabla u|}^{\prime}(|\nabla u-\nabla v|)|\nabla u-\nabla v| d x \sim \int_{\Omega} \varphi_{|\nabla u|}(|\nabla u-\nabla v|) d x .
\end{aligned}
$$

This ensures in case of the p-Laplacian that all the results below can also be expressed in terms of the quasi-norm.

Addtionally, we need the following direct consequence of Lemma 3:

Corollary 6. Let $\mathbf{A}, \varphi, \mathbf{F}$ be as in Lemma 3. Then for all $\mathbf{a}, \mathbf{b} \in \mathbb{R}^{d}$

$$
\left(\varphi_{|\mathbf{a}|}\right)^{*}(|\mathbf{A}(\mathbf{a})-\mathbf{A}(\mathbf{b})|) \sim \varphi_{|\mathbf{a}|}(|\mathbf{a}-\mathbf{b}|) \sim|\mathbf{F}(\mathbf{a})-\mathbf{F}(\mathbf{b})|^{2} .
$$

Proof. The second relation is contained in Lemma 3. The first relation follows from (3.3d), (2.6), and $\Delta_{2}(\varphi)<\infty$. 
3.2. Finite element spaces. Let $\mathcal{T}_{H}$ be a conforming triangulation of $\Omega$ consisting of closed simplices $T \in \mathcal{T}_{H}$. Let $h_{T}$ denote the diameter of the (closed) simplex $T \in \mathcal{T}_{H}$ and $\rho_{T}$ the maximal radius of a ball that is contained in $T$. The maximal quotient $h_{T} / \rho_{T}$ with $T \in \mathcal{T}_{h}$ is called the shape regularity (chunkiness) of $\mathcal{T}_{H}$.

Let $V_{H}:=V\left(\mathcal{T}_{H}\right)$ be the space of continuous, piecewise linear finite elements over $\mathcal{T}_{H}$ with boundary values zero, then $V_{H} \subset V$. By $u_{H} \in V_{H}$ we denote the finite element solution of $(2.9)$ with respect to $V_{H}$, i.e.

$$
\left\langle\mathbf{A}\left(\nabla u_{H}\right), \nabla v_{H}\right\rangle=\left\langle f, v_{H}\right\rangle \quad \text { for all } v_{H} \in V_{H},
$$

where $\left\langle f, v_{H}\right\rangle=\int_{\Omega} f v_{H} d x$. The theory of monotone operators ensures the existence of a unique solution.

We denote by $\Gamma_{H}$ the set of interior faces (sides) of the triangulation $\mathcal{T}_{H}$. For $\gamma \in \Gamma_{H}$ we define $N_{\gamma}$ as the set of elements sharing $\gamma$ and $S_{\gamma}$ as the union of these elements, i.e.

$$
N_{\gamma}:=\left\{T_{1}, T_{2} \in \mathcal{T}_{H}: T_{1} \cap T_{2}=\gamma\right\}, \quad S_{\gamma}:=\bigcup_{T \in N_{\gamma}} T .
$$

For $T \in \mathcal{T}_{H}$ define the set of neighbours $N_{T}$ and the neighbourhood $S_{T}$ by

$$
N_{T}:=\left\{T^{\prime} \in \mathcal{T}_{H}: T^{\prime} \cap T \in \Gamma_{H}\right\}, \quad S_{T}:=\bigcup_{T^{\prime} \in N_{T}} T^{\prime} .
$$

For interpolation estimates (see (3.6)) we additionally need to define the patch $\Omega_{T}$ around $T$ and the set of its elements $\omega_{T}$ by

$$
\omega_{T}:=\left\{T^{\prime} \in \mathcal{T}_{H}: T^{\prime} \cap T \neq \emptyset\right\}, \quad \Omega_{T}:=\bigcup_{T^{\prime} \in \omega_{T}} T^{\prime} .
$$

For $\gamma \in \Gamma_{H}$ let $h_{\gamma}:=\operatorname{diam}(\gamma)$. For $T \in \mathcal{T}_{H}$ holds $h_{T} \sim h_{\gamma}$ for each face (side) $\gamma \subset \partial T$ depending only on the shape regularity of $\mathcal{T}_{H}$.

Let $\Pi_{H}: V \rightarrow V_{H}$ be the Scott-Zhang interpolation operator which respects zero boundary values, see [SZ90]. Additionally $\Pi_{H}$ satisfies $\Pi_{H} v_{H}=v_{H}$ for all $v_{H} \in V_{H}$. It was shown in [DR06] that for all $v \in W^{1, \varphi}(\Omega), a \geq 0$, and $T \in \mathcal{T}_{h}$

$$
\int_{T} \varphi_{a}\left(\left|v-\Pi_{H} v\right|\right) d x+\int_{T} \varphi_{a}\left(h_{T}\left|\nabla v-\nabla \Pi_{H} v\right|\right) d x \leq C \int_{\Omega_{T}} \varphi_{a}\left(h_{T}|\nabla v|\right) d x
$$

where $C$ only depends on $\Delta_{2}(\varphi)$ and the shape regularity of $\mathcal{T}_{H}$.

We introduce residual based error estimators for our system (2.1). For $\gamma \in \Gamma_{H}$ and $T \in \mathcal{T}_{H}$ define the (local) interior and the jump estimators by

$$
\begin{aligned}
\eta_{E}^{2}\left(u_{H}, T\right) & :=\int_{T}\left(\varphi_{\left|\nabla u_{H}\right|}\right)^{*}\left(h_{T}|f|\right) d x, \\
\eta_{J}^{2}\left(u_{H}, \gamma\right) & :=\int_{\gamma} h_{\gamma}\left|\llbracket \mathbf{F}\left(\nabla u_{H}\right) \rrbracket_{\gamma}\right|^{2} d x .
\end{aligned}
$$

where $\llbracket \mathbf{F}\left(\nabla u_{H}\right) \rrbracket_{\gamma}$ denotes the jump of $\mathbf{F}\left(\nabla u_{H}\right)$ over the face $\gamma$. Furthermore we define for $T \in \mathcal{T}_{H}$ the (local) element based error indicators and the oscillation as

$$
\begin{aligned}
\eta^{2}\left(u_{H}, T\right) & :=\eta_{E}^{2}\left(u_{H}, T\right)+\sum_{\gamma \in \Gamma_{H}, \gamma \subset \partial T} \eta_{J}^{2}\left(u_{H}, \gamma\right), \\
\operatorname{osc}^{2}\left(u_{H}, T\right) & :=\inf _{f_{T} \in \mathbb{R}} \int_{T}\left(\varphi_{\left|\nabla u_{H}\right|}\right)^{*}\left(h_{T}\left|f-f_{T}\right|\right) d x .
\end{aligned}
$$

For a subset $\hat{\mathcal{T}}_{H} \subset \mathcal{T}_{H}$ we define the total error estimator over $\hat{\mathcal{T}}_{H}$ by

$$
\eta^{2}\left(u_{k}, \hat{\mathcal{T}}_{H}\right):=\sum_{T \in \hat{\mathcal{T}}_{H}} \eta^{2}\left(u_{k}, T\right)
$$


Note that $\eta^{2}\left(u_{H},\{T\}\right)=\eta^{2}\left(u_{H}, T\right)$, so there is no confusion between this definition of $\eta^{2}$ and (3.7). Similarly, we define the total oscillation on subsets of $\mathcal{T}_{H}$.

Furthermore we use $e_{H}:=u-u_{H}$ for the difference of the solutions.

Remark 7. In the case of the p-Laplacian we can translate the above definitions of the estimators and the oscillation. For the jump estimator we obtain by Corollary 4 , Lemma 22, and Remark 5

$$
\begin{aligned}
\eta_{J}^{2}\left(u_{H}, \gamma\right) & =\int_{\gamma} h_{\gamma}\left|\llbracket \mathbf{F}\left(\nabla u_{H}\right) \rrbracket_{\gamma}\right|^{2} d x \sim \int_{S_{\gamma}} \varphi_{\left|\nabla u_{H}\right|}\left(\llbracket \nabla u_{H} \rrbracket_{\gamma}\right) \\
& \sim \int_{S_{\gamma}}\left(\kappa+\left|\nabla u_{H}\right|+\left|\llbracket \nabla u_{H} \rrbracket_{\gamma}\right|\right)^{p-2}\left|\llbracket \nabla u_{H} \rrbracket_{\gamma}\right| d x .
\end{aligned}
$$

The element residual an the oscillation can be treated in the same way. We use Lemma 22, (2.6), and Remark 5 to obtain

$$
\begin{aligned}
\eta_{E}^{2}\left(u_{H}, T\right) & =\int_{T}\left(\varphi\left|\nabla u_{H}\right|\right)^{*}\left(h_{T}|f|\right) d x \sim \int_{T}\left(\varphi^{*}\right)_{\varphi^{\prime}\left(\left|\nabla u_{H}\right|\right)}\left(h_{T}|f|\right) d x \\
& \sim \int_{T}\left(\kappa+\left|\nabla u_{H}\right|^{p-1}+h_{T}|f|\right)^{q-2} h_{T}^{2}|f|^{2} d x .
\end{aligned}
$$

Let $f_{T}$ denote the mean value of $f$ over $T$. Then the same calculations yield for the oscillation

$$
\operatorname{osc}^{2}\left(u_{H}, T\right) \sim \int_{T}\left(\kappa+\left|\nabla u_{H}\right|^{p-1}+h_{T}\left|f-f_{T}\right|\right)^{q-2} h_{T}^{2}\left|f-f_{T}\right|^{2} d x,
$$

where we have used that for any $N$-function $\varrho$ with $\Delta_{2}(\varrho)<\infty$ holds

$$
\inf _{f_{T}} \int_{T} \varrho\left(\left|f-f_{T}\right|\right) d x \leq \int_{T} \varrho\left(\left|f-f_{T}\right|\right) d x \leq c \inf _{f_{T}} \int_{T} \varrho\left(\left|f-f_{T}\right|\right) d x
$$

with constants c only depending on $\Delta_{2}(\varphi)$. Thus our a posteriori estimators improve the one in [LY02].

3.3. Upper Bound. To obtain the upper bound we use Lemma 3, the Galerkin orthogonality, and $\Pi_{H}: V \rightarrow V_{H}$ :

$$
\begin{aligned}
\left\|\mathbf{F}\left(\nabla u_{H}\right)-\mathbf{F}(\nabla u)\right\|_{2}^{2} & \sim\left\langle\mathbf{A}(\nabla u)-\mathbf{A}\left(\nabla u_{H}\right), \nabla\left(u-u_{H}\right)\right\rangle \\
& =\left\langle\mathbf{A}(\nabla u)-\mathbf{A}\left(\nabla u_{H}\right), \nabla\left(e_{H}-\Pi_{H} e_{H}\right)\right\rangle .
\end{aligned}
$$

By integration by parts on each $T \in \mathcal{T}_{h}$ we get

$$
\begin{aligned}
\| \mathbf{F} & \left(\nabla u_{H}\right)-\mathbf{F}(\nabla u) \|_{2}^{2} \\
& \sim-\sum_{T \in \mathcal{T}_{H}} \int_{\partial T}\left(\mathbf{A}\left(\nabla u_{H}\right) \cdot \mathbf{n}\right)\left(e_{H}-\Pi_{H} e_{H}\right) d x+\left\langle f, e_{H}-\Pi_{H} e_{H}\right\rangle \\
& =-\sum_{\gamma \in \Gamma_{H}} \int_{\gamma} \llbracket \mathbf{A}\left(\nabla u_{H}\right) \cdot \mathbf{n} \rrbracket_{\gamma}\left(e_{H}-\Pi_{H} e_{H}\right) d x+\left\langle f, e_{H}-\Pi_{H} e_{H}\right\rangle \\
& =\left(\text { Upper }_{1}\right)+\left(\text { Upper }_{2}\right),
\end{aligned}
$$

where $\mathbf{n}$ is the outernormal of $\partial T$. We handle the two terms jump residual (Upper ${ }_{1}$ ) and element residual (Upper 2 ) separately. First we estimate

$$
\text { Upper } \left._{1}\right) \leq \sum_{\gamma \in \Gamma_{H}}\left|\llbracket \mathbf{A}\left(\nabla u_{H}\right) \rrbracket_{\gamma}\right| \int_{\gamma}\left|e_{H}-\Pi_{H} e_{H}\right| d x
$$


where we have used that $\nabla u_{H}$ is constant on each $T \in \mathcal{T}_{H}$. The trace theorem $W^{1,1}\left(S_{\gamma}\right) \hookrightarrow L^{1}(\gamma)$ and the $W^{1,1}$-approximability of $\Pi_{H}$ (see [SZ90] or use (3.6) with $\varphi(t)=t$ and $a=0)$ imply

$$
\begin{aligned}
\int_{\gamma}\left|e_{H}-\Pi_{H} e_{H}\right| d x & \leq C h_{\gamma} \int_{S_{\gamma}}\left|e_{H}-\Pi_{H} e_{H}\right| d x+C \int_{S_{\gamma}}\left|\nabla e_{H}-\nabla \Pi_{H} e_{H}\right| d x \\
& \leq c \sum_{T \in N_{\gamma}} \int_{\Omega_{T}}\left|\nabla e_{h}\right| d x .
\end{aligned}
$$

If we combine this with (3.9), then we get

$$
\text { (Upper } \left._{1}\right) \leq C \sum_{\gamma \in \Gamma_{H}}\left(\left|\llbracket \mathbf{A}\left(\nabla u_{H}\right) \rrbracket_{\gamma}\right| \sum_{T \in N_{\gamma}} \int_{\Omega_{T}}\left|\nabla e_{H}\right| d x\right) .
$$

Now Young's inequality (2.3) for $\varphi_{\left|\nabla u_{H}\right|}$ on each element yields

$$
\text { Upper } \left._{1}\right) \leq \sum_{\gamma \in \Gamma_{H}} \sum_{T \in N_{\gamma}} \int_{\Omega_{T}} C_{\delta}\left(\varphi_{\left|\nabla u_{H}\right|}\right)^{*}\left(\left|\llbracket \mathbf{A}\left(\nabla u_{H}\right) \rrbracket_{\gamma}\right|\right)+\delta \varphi_{\left|\nabla u_{H}\right|}\left(\left|\nabla e_{H}\right|\right) d x
$$

Due to (3.4) we have

$$
\left(\varphi_{\left|\nabla u_{H}\right|}\right)^{*}\left(\left|\llbracket \mathbf{A}\left(\nabla u_{H}\right) \rrbracket_{\gamma}\right|\right) \sim \varphi_{\left|\nabla u_{H}\right|}\left(\left|\llbracket \nabla u_{H} \rrbracket_{\gamma}\right|\right) \sim\left|\llbracket \mathbf{F}\left(\nabla u_{H}\right) \rrbracket_{\gamma}\right|^{2} .
$$

Note that due to shape regularity it holds $h_{T^{\prime}} \sim h_{T} \sim h_{\gamma}$ for $T^{\prime} \in \omega_{T}, T \in N_{\gamma}$. With the help of (3.10), Lemma 3, the finite overlapping of the $\Omega_{T}$, and $\# N_{T}=2$ we get

$$
\begin{aligned}
\text { Upper } \left._{1}\right) \leq & C_{\delta} C \sum_{\gamma \in \Gamma_{H}} \sum_{T \in N_{\gamma}} \int_{\Omega_{T}}\left|\llbracket \mathbf{F}\left(\nabla u_{H}\right) \rrbracket_{\gamma}\right|^{2} \\
& +\delta C \sum_{\gamma \in \Gamma_{H}} \sum_{T \in N_{\gamma}} \int_{\Omega_{T}}\left|\mathbf{F}(\nabla u)-\mathbf{F}\left(\nabla u_{H}\right)\right|^{2} d x \\
\leq & C_{\delta} C \sum_{\gamma \in \Gamma_{H}} \int_{\gamma} h_{\gamma}\left|\llbracket \mathbf{F}\left(\nabla u_{H}\right) \rrbracket_{\gamma}\right|^{2} \\
& +\delta C \sum_{\gamma \in \Gamma_{H}} \sum_{T \in N_{\gamma}} \int_{\Omega_{T}}\left|\mathbf{F}(\nabla u)-\mathbf{F}\left(\nabla u_{H}\right)\right|^{2} d x \\
\leq & C_{\delta} C \sum_{\gamma \in \Gamma_{H}} \eta_{J}^{2}\left(u_{H}, \gamma\right)+\delta C\left\|\mathbf{F}\left(\nabla u_{H}\right)-\mathbf{F}(\nabla u)\right\|_{2}^{2} .
\end{aligned}
$$

We treat the element residual as follows

$$
\begin{aligned}
\text { (Upper } \left._{2}\right) & \leq \sum_{T \in \mathcal{T}_{H}} \int_{T}|f|\left|e_{H}-\Pi_{H} e_{H}\right| d x \\
& \leq \sum_{T \in \mathcal{T}_{H}} \int_{T} C_{\delta}\left(\varphi_{\left|\nabla u_{H}\right|}\right)^{*}\left(h_{T}|f|\right)+\delta\left(\varphi_{\left|\nabla u_{H}\right|}\right)\left(\frac{\left|e_{H}-\Pi_{H} e_{H}\right|}{h_{T}}\right) d x,
\end{aligned}
$$

where we have used Young's inequality (2.3). Since $\nabla u_{H}$ is constant on each $T \in \mathcal{T}_{H}$ we can use (3.6) to obtain

$$
\text { (Upper } \left._{2}\right) \leq C_{\delta} C \sum_{T \in \mathcal{T}_{H}} \eta_{E}^{2}\left(u_{H}, T\right)+\delta C \sum_{T \in \mathcal{T}_{H}} \int_{\Omega_{T}} \varphi_{\left|\nabla u_{H}(T)\right|}\left(\left|\nabla e_{H}\right|\right) d x,
$$

where we write $\nabla u_{H}(T)$ to indicate that the shift on the whole $\Omega_{T}$ depends on the value of $\nabla u_{H}$ on the triangle $T$. In order to get $\varphi_{\left|\nabla u_{H}\right|}\left(\left|\nabla e_{H}\right|\right)$ instead of 
$\varphi_{\left|\nabla u_{H}(T)\right|}\left(\left|\nabla e_{H}\right|\right)$ we need a change of shift. We apply Corollary 26 on each $T^{\prime} \in \omega_{T}$ and get

$$
\begin{aligned}
\text { Upper } \left._{2}\right) \leq & C_{\delta} C \sum_{T \in \mathcal{T}_{H}} \eta_{E}^{2}\left(u_{H}, T\right)+\delta C \sum_{T \in \mathcal{T}_{H}} \int_{\Omega_{T}} \varphi_{\left|\nabla u_{H}\right|}\left(\left|\nabla e_{H}\right|\right) d x \\
& +\delta C \sum_{T \in \mathcal{T}_{H}} \sum_{T^{\prime} \in \omega_{T}} \int_{T^{\prime}}\left|\mathbf{F}\left(\nabla u_{H}(T)\right)-\mathbf{F}\left(\nabla u_{H}\left(T^{\prime}\right)\right)\right|^{2} d x
\end{aligned}
$$

Now we transform the last term. Since one can reach $T^{\prime}$ from $T$ by passing through a finite number of faces (depending on the shape regularity of $\mathcal{T}_{H}$ ), we can estimate each $\left|\mathbf{F}\left(\nabla u_{H}(T)\right)-\mathbf{F}\left(\nabla u_{H}\left(T^{\prime}\right)\right)\right|$ for $T^{\prime} \in \omega_{T}$ by a sum of jumps $\left|\llbracket \mathbf{F}\left(\nabla u_{H}\right) \rrbracket_{\gamma}\right|$ over these faces. In particular,

$$
\begin{gathered}
\sum_{T \in \mathcal{T}_{H}} \sum_{T^{\prime} \in \omega_{T}} \int_{T^{\prime}}\left|\mathbf{F}\left(\nabla u_{H}(T)\right)-\mathbf{F}\left(\nabla u_{H}\left(T^{\prime}\right)\right)\right|^{2} d x \sim \sum_{\gamma \in \Gamma_{H}} \int_{S_{\gamma}}\left|\llbracket \mathbf{F}\left(\nabla u_{H}\right) \rrbracket_{\gamma}\right|^{2} d x \\
\sim \sum_{\gamma \in \Gamma_{H}} h_{\gamma} \int_{\gamma}\left|\llbracket \mathbf{F}\left(\nabla u_{H}\right) \rrbracket_{\gamma}\right|^{2} d x=\sum_{\gamma \in \Gamma_{H}} \eta_{J}^{2}\left(u_{H}, \gamma\right)
\end{gathered}
$$

using that $\nabla u_{H}$ is piecewise constant and $\left|S_{\gamma}\right| \sim h_{\gamma}|\gamma|$. Hence, with Lemma 3

$$
\begin{aligned}
& \left(\text { Upper }_{2}\right) \\
& \leq C_{\delta} C \sum_{T \in \mathcal{T}_{H}} \eta_{E}^{2}\left(u_{H}, T\right)+\delta C \sum_{T \in \mathcal{T}_{H}} \int_{\Omega_{T}} \varphi_{\left|\nabla u_{H}\right|}\left(\left|\nabla e_{H}\right|\right) d x+\delta C \sum_{\gamma \in \Gamma_{H}} \eta_{J}^{2}\left(u_{H}, \gamma\right) \\
& \leq C_{\delta} C \sum_{T \in \mathcal{T}_{H}} \eta_{E}^{2}\left(u_{H}, T\right)+\delta C\left\|\mathbf{F}\left(\nabla u_{H}\right)-\mathbf{F}(\nabla u)\right\|_{2}^{2}+\delta C \sum_{\gamma \in \Gamma_{H}} \eta_{J}^{2}\left(u_{H}, \gamma\right) .
\end{aligned}
$$

Now, taking $\delta>0$ small enough we obtain from (3.8), (3.11), the last inequality, (3.7) and the fact that each side $\gamma \in \Gamma_{H}$ is shared by at most two elements:

Lemma 8 (Upper Bound). For finite element solutions $u_{H}$ of (3.5) it holds

$$
\left\|\mathbf{F}\left(\nabla u_{H}\right)-\mathbf{F}(\nabla u)\right\|_{2}^{2} \leq C \eta^{2}\left(u_{H}, \mathcal{T}_{H}\right),
$$

where the constant $C$ only depend on $\Delta_{2}(\varphi), \Delta_{2}\left(\varphi^{*}\right)$, and the shape regularity of $\mathcal{T}_{H}$.

3.4. Lower Bound. In this section we show that the error can be locally estimated from below by the error estimators. We begin with the element estimator. As is well known, for each $T \in \mathcal{T}_{H}$ there exists a bubble function $w_{T} \in W_{0}^{1, \varphi}(T)$ with $w_{T} \geq 0$ and

$$
\int_{T} w_{T} d x=|T|, \quad\left\|w_{T}\right\|_{\infty} \leq C, \quad\left\|\nabla w_{T}\right\|_{\infty} \leq \frac{C}{h_{T}},
$$

where $C>0$ depends only on the shape regularity of $\mathcal{T}_{H}$. Then for $s \in \mathbb{R}$

$$
\left\langle\mathbf{A}(\nabla u)-\mathbf{A}\left(\nabla u_{H}\right), \nabla\left(s w_{T}\right)\right\rangle=\left\langle f, s w_{T}\right\rangle,
$$

where we have used that $\nabla u_{H}$ is constant on $T$. For $f_{T} \in \mathbb{R}$ by (2.4) applied to $\varphi_{\left|\nabla u_{H}\right|}$ there exists $s_{T} \in \mathbb{R}$ such that

$$
s_{T}\left(h_{T} f_{T}\right)=\left(\varphi_{\left|\nabla u_{H}(T)\right|}\right)^{*}\left(h_{T}\left|f_{T}\right|\right)+\varphi_{\left|\nabla u_{H}\right|}\left(\left|s_{T}\right|\right),
$$

i.e. Young's inequality is sharp. We obtain with (3.15) and (3.14) taking $s=h_{T} s_{T}$

$$
\begin{aligned}
|T| & \left(\varphi_{\left|\nabla u_{H}(T)\right|}\right)^{*}\left(h_{T}\left|f_{T}\right|\right)+|T| \varphi_{\left|\nabla u_{H}(T)\right|}\left(\left|s_{T}\right|\right)=|T| f_{T} h_{T} s_{T} \\
\quad & =\left\langle\mathbf{A}(\nabla u)-\mathbf{A}\left(\nabla u_{H}(T)\right), \nabla\left(h_{T} s_{T} w_{T}\right)\right\rangle+\left\langle f_{T}-f, h_{T} s_{T} w_{T}\right\rangle \\
& =\left(\text { Lower }_{1}\right)+\left(\text { Lower }_{2}\right) .
\end{aligned}
$$


With the help of (3.13), Young's inequality (2.3), (3.4) and the fact that the integrant is constant $\varphi_{\left|\nabla u_{H}\right|}\left(\left|s_{T}\right|\right)=\varphi_{\left|\nabla u_{H}(T)\right|}\left(\left|s_{T}\right|\right)$ on $T$, we have

$$
\begin{aligned}
\text { Lower } \left._{1}\right) & \leq C \int_{T}\left|\mathbf{A}(\nabla u)-\mathbf{A}\left(\nabla u_{H}\right)\right|\left|s_{T}\right| d x \\
& \leq C_{\delta} C \int_{T}\left|\mathbf{F}(\nabla u)-\mathbf{F}\left(\nabla u_{H}\right)\right|^{2} d x+\delta C|T| \varphi_{\left|\nabla u_{H}(T)\right|}\left(\left|s_{T}\right|\right) .
\end{aligned}
$$

Similarly, with (3.13) and Young's inequality (2.3) we get

$$
\begin{aligned}
\left(\text { Lower }_{2}\right) & \leq C \int_{T} h_{T} s_{T}\left|f-f_{T}\right| d x \\
& \leq C_{\delta} C \int_{T}\left(\varphi_{\left|\nabla u_{H}\right|}\right)^{*}\left(h_{T}\left|f-f_{T}\right|\right) d x+\delta C|T| \varphi_{\left|\nabla u_{H}(T)\right|}\left(\left|s_{T}\right|\right) .
\end{aligned}
$$

Now, taking $\delta>0$ small enough we obtain from (3.16), (3.17), and (3.18) that

$$
\begin{aligned}
|T|\left(\varphi_{\left|\nabla u_{H}(T)\right|}\right)^{*}\left(h_{T}\left|f_{T}\right|\right) \leq & C \int_{T}\left|\mathbf{F}(\nabla u)-\mathbf{F}\left(\nabla u_{H}\right)\right|^{2} d x \\
& +C \int_{T}\left(\varphi_{\left|\nabla u_{H}\right|}\right)^{*}\left(h_{T}\left|f-f_{T}\right|\right) d x .
\end{aligned}
$$

Observe that by convexity of $\left(\varphi_{\left|\nabla u_{H}(T)\right|}\right)^{*}, \Delta_{2}\left(\varphi^{*}\right), \Delta_{2}(\varphi)<\infty$ and Lemma 22

$$
\begin{aligned}
\eta_{E}^{2}\left(u_{H}, T\right) & =\int_{T}\left(\varphi_{\left|\nabla u_{H}\right|}\right)^{*}\left(h_{T}|f|\right) \\
& \leq \int_{T}\left(\varphi_{\left|\nabla u_{H}\right|}\right)^{*}\left(2 h_{T}\left|f-f_{T}\right|\right)+\left(\varphi_{\left|\nabla u_{H}\right|}\right)^{*}\left(2 h_{T}\left|f_{T}\right|\right) \\
& \leq C \int_{T}\left(\varphi_{\left|\nabla u_{H}\right|}\right)^{*}\left(h_{T}\left|f-f_{T}\right|\right) d x+C|T|\left(\varphi_{\left|\nabla u_{H}(T)\right|}\right)^{*}\left(h_{T}\left|f_{T}\right|\right)
\end{aligned}
$$

with $C>0$ depending only on $\Delta_{2}(\varphi)$ and $\Delta_{2}\left(\varphi^{*}\right)$. This and (3.19) gives

$$
\eta_{E}^{2}\left(u_{H}, T\right) \leq C \int_{T}\left|\mathbf{F}(\nabla u)-\mathbf{F}\left(\nabla u_{H}\right)\right|^{2} d x+C \int_{T}\left(\varphi\left|\nabla u_{H}\right|\right)^{*}\left(h_{T}\left|f-f_{T}\right|\right) d x .
$$

Taking the infimum over all $f_{T} \in \mathbb{R}$ proves the following assertion:

Lemma 9. For finite element solutions $u_{H}$ of (3.5) and $T \in \mathcal{T}_{h}$ it holds

$$
\eta_{E}^{2}\left(u_{H}, T\right) \leq C\left\|\mathbf{F}\left(\nabla u_{H}\right)-\mathbf{F}(\nabla u)\right\|_{L^{2}(T)}^{2}+C \operatorname{osc}^{2}\left(u_{H}, T\right) .
$$

where the constant $C$ only depends on $\Delta_{2}(\varphi), \Delta_{2}\left(\varphi^{*}\right)$, and the shape regularity of $\mathcal{T}_{H}$.

Now we estimate the jump estimator. As is well known, for each $\gamma \in \Gamma_{H}$ there exists a bubble function $w_{\gamma} \in W_{0}^{1, \varphi}\left(S_{\gamma}\right)$ with $w_{\gamma} \geq 0$ and

$$
\int_{\gamma} w_{\gamma} d x=|\gamma|, \quad\left\|w_{\gamma}\right\|_{\infty} \leq C, \quad\left\|\nabla w_{\gamma}\right\|_{\infty} \leq \frac{C}{h_{\gamma}},
$$

where $C>0$ depends only on the shape regularity of $\mathcal{T}_{H}$. Then for $s \in \mathbb{R}$

$$
\begin{aligned}
\left\langle\mathbf{A}(\nabla u)-\mathbf{A}\left(\nabla u_{H}\right), \nabla\left(s w_{\gamma}\right)\right\rangle & =\left\langle f, s w_{\gamma}\right\rangle-\int_{\gamma} \llbracket \mathbf{A}\left(\nabla u_{H}\right) \cdot \mathbf{n} \rrbracket_{\gamma} s w_{\gamma} d x \\
& =\left\langle f, s w_{\gamma}\right\rangle-|\gamma| \llbracket \mathbf{A}\left(\nabla u_{H}\right) \cdot \mathbf{n} \rrbracket_{\gamma} s,
\end{aligned}
$$


where we have used partial integration and that $\nabla u_{H}$ is piecewise constant. Let $T_{0}$, $T_{1}$ be the two triangles sharing $\gamma$. Then by (2.4) applied to $\varphi_{\left|\nabla u_{H}\left(T_{0}\right)\right|}$ there exists $s_{\gamma} \in \mathbb{R}$ such that

$$
\llbracket \mathbf{A}\left(\nabla u_{H}\right) \cdot \mathbf{n} \rrbracket_{\gamma} s_{\gamma}=\left(\varphi_{\left|\nabla u_{H}\left(T_{0}\right)\right|}\right)^{*}\left(\left|\llbracket \mathbf{A}\left(\nabla u_{H}\right) \cdot \mathbf{n} \rrbracket_{\gamma}\right|\right)+\varphi_{\left|\nabla u_{H}\left(T_{0}\right)\right|}\left(\left|s_{\gamma}\right|\right),
$$

i.e. Young's inequality is sharp. We have chosen $\left|\nabla u_{H}\left(T_{0}\right)\right|$ as the shift, which puts $T_{0}$ into a special position, but we will see later that it is not important which of the two triangles is chosen. Let $s=\frac{\left|S_{\gamma}\right|}{|\gamma|} s_{\gamma}$ in (3.23), then we obtain with (3.24)

$$
\begin{aligned}
\left|S_{\gamma}\right| & \left(\varphi_{\left|\nabla u_{H}\left(T_{0}\right)\right|}\right)^{*}\left(\llbracket \mathbf{A}\left(\nabla u_{H}\right) \cdot \mathbf{n} \rrbracket_{\gamma}\right)+\left|S_{\gamma}\right| \varphi_{\left|\nabla u_{H}\left(T_{0}\right)\right|}\left(\left|s_{\gamma}\right|\right) \\
& =|S \gamma| \llbracket \mathbf{A}\left(\nabla u_{H}\right) \cdot \mathbf{n} \rrbracket_{\gamma} s_{\gamma} \\
& =-\frac{\left|S_{\gamma}\right|}{|\gamma|}\left\langle\mathbf{A}(\nabla u)-\mathbf{A}\left(\nabla u_{H}\right), \nabla\left(s_{\gamma} w_{\gamma}\right)\right\rangle+\frac{\left|S_{\gamma}\right|}{|\gamma|}\left\langle f, s_{\gamma} w_{\gamma}\right\rangle \\
& =\left(\text { Lower }_{3}\right)+\left(\text { Lower }_{4}\right) .
\end{aligned}
$$

Before we proceed with the estimates for $\left(\right.$ Lower $\left._{3}\right)$ and $\left(\right.$ Lower $\left._{4}\right)$ we simplify the $\operatorname{term}\left(\varphi\left|\nabla u_{H}\left(T_{0}\right)\right|\right)^{*}\left(\left|\llbracket \mathbf{A}\left(\nabla u_{H}\right) \cdot \mathbf{n} \rrbracket_{\gamma}\right|\right)$. First we show that

$$
\left|\llbracket \mathbf{A}\left(\nabla u_{H}\right) \cdot \mathbf{n} \rrbracket_{\gamma}\right| \sim \varphi_{\left|\nabla u_{H}\right|}^{\prime}\left(\left|\llbracket \nabla u_{H} \rrbracket_{\gamma}\right|\right) \sim\left|\llbracket \mathbf{A}\left(\nabla u_{H}\right) \rrbracket_{\gamma}\right| .
$$

The last part of (3.26) is an immediate consequence of Lemma 3. If $\llbracket \nabla u_{H} \rrbracket_{\gamma}=0$, then also $\llbracket \mathbf{A}\left(\nabla u_{H}\right) \cdot \mathbf{n} \rrbracket_{\gamma}=0$ and all terms in (3.26) are zero. So we can assume $\llbracket \nabla u_{H} \rrbracket_{\gamma} \neq 0$. Since $u_{H} \in C\left(S_{\gamma}\right)$, the tangential derivatives of $u_{H}$ are continuous on $\gamma$ and do not jump. Hence, $\left|\llbracket \nabla u_{H} \rrbracket_{\gamma}\right|=\left|\llbracket \nabla u_{H} \rrbracket_{\gamma} \cdot \mathbf{n}\right|$ and

$$
\mathbf{n}= \pm \frac{\llbracket \nabla u_{H} \rrbracket_{\gamma}}{\left|\llbracket \nabla u_{H} \rrbracket_{\gamma}\right|} .
$$

This and (3.3) imply

$$
\left|\llbracket \mathbf{A}\left(\nabla u_{H}\right) \cdot \mathbf{n} \rrbracket_{\gamma}\right| \cdot\left|\llbracket \nabla u_{H} \rrbracket_{\gamma}\right|=\left|\llbracket \mathbf{A}\left(\nabla u_{H}\right) \rrbracket_{\gamma} \cdot \llbracket \nabla u_{H} \rrbracket_{\gamma}\right| \sim \varphi_{\left|\nabla u_{H}\right|}\left(\left|\llbracket \nabla u_{H} \rrbracket_{\gamma}\right|\right) .
$$

Now, (2.6) proves (3.26). Lemma 22 states that $\left(\varphi_{\left|u_{H}\right|}\right)^{*}$ is an N-function with $\Delta_{2}\left(\left(\varphi_{\left|u_{H}\right|}\right)^{*}\right)<\infty$ depending solely on $\Delta_{2}(\varphi)$ and $\Delta_{2}\left(\varphi^{*}\right)$. Therefor we get with (3.4), and (3.26), and

$$
\begin{aligned}
\left(\varphi_{\left|\nabla u_{H}\left(T_{0}\right)\right|}\right)^{*}\left(\left|\llbracket \mathbf{A}\left(\nabla u_{H}\right) \cdot \mathbf{n} \rrbracket_{\gamma}\right|\right) & \sim\left(\varphi_{\left|\nabla u_{H}\left(T_{0}\right)\right|}\right)^{*}\left(\left|\llbracket \mathbf{A}\left(\nabla u_{H}\right) \rrbracket_{\gamma}\right|\right) \\
& \sim\left|\llbracket \mathbf{F}\left(\nabla u_{H}\right) \rrbracket_{\gamma}\right|^{2} .
\end{aligned}
$$

Now, it becomes clear, why the preference of $T_{0}$ is not important: The expression $\left|\llbracket \mathbf{F}\left(\nabla u_{H}\right) \rrbracket_{\gamma}\right|^{2}$ in (3.27) is symmetrical in $T_{0}$ and $T_{1}$ and therefor independent of the choice $T_{0}$.

We proceed with the estimate for (Lower 3 ). With $\left|S_{\gamma}\right| \sim h_{\gamma}|\gamma|,(3.22)$, Young's inequality (2.3), and (3.4) we get

$$
\begin{aligned}
\left(\text { Lower }_{3}\right) & \leq C \int_{S_{\gamma}}\left|\mathbf{A}(\nabla u)-\mathbf{A}\left(\nabla u_{H}\right)\right|\left|s_{\gamma}\right| d x \\
& \leq C_{\delta} C \int_{S_{\gamma}}\left|\mathbf{F}(\nabla u)-\mathbf{F}\left(\nabla u_{H}\right)\right|^{2} d x+\delta C \sum_{T \in N_{\gamma}}|T| \varphi_{\left|\nabla u_{H}(T)\right|}\left(\left|s_{\gamma}\right|\right) .
\end{aligned}
$$


With $\left|S_{\gamma}\right| \sim h_{\gamma}|\gamma|$, (3.22), and Young's inequality (2.3) we deduce

$$
\begin{aligned}
& \left(\text { Lower }_{4}\right) \\
& \leq C \int_{S_{\gamma}}|f| h_{\gamma}\left|s_{\gamma}\right| d x \\
& \leq C \sum_{T \in N_{\gamma}} \inf _{f_{T} \in \mathbb{R}} \int_{T}\left|f-f_{T}\right| h_{\gamma}\left|s_{\gamma}\right|+\left|f_{T}\right| h_{\gamma}\left|s_{\gamma}\right| d x \\
& \leq C_{\delta} C \sum_{T \in N_{\gamma}} \inf _{f_{T} \in \mathbb{R}} \int_{T}\left(\varphi\left|\nabla u_{H}\right|\right)^{*}\left(h_{\gamma}\left|f-f_{T}\right|\right) d x+\left(\varphi\left|\nabla u_{H}\right|\right)^{*}\left(h_{\gamma}\left|f_{T}\right|\right) d x \\
& \quad+\delta C \sum_{T \in N_{\gamma}} \int_{T} \varphi_{\left|\nabla u_{H}\right|}\left(\left|s_{\gamma}\right|\right) d x \\
& \leq C_{\delta} C \sum_{T \in N_{\gamma}} \operatorname{osc}^{2}\left(u_{H}, T\right)+C_{\delta} C \sum_{T \in N_{\gamma}} \eta_{E}^{2}\left(u_{H}, T\right)+\delta C \sum_{T \in N_{\gamma}}|T| \varphi_{\left|\nabla u_{H}(T)\right|}\left(\left|s_{\gamma}\right|\right) .
\end{aligned}
$$

This, (3.25), (3.28), and $\left|T_{0}\right|,\left|T_{1}\right| \leq\left|S_{\gamma}\right|$ imply

$$
\begin{aligned}
& \left|S_{\gamma}\right|\left(\varphi_{\left|\nabla u_{H}\left(T_{0}\right)\right|}\right)^{*}\left(\left|\llbracket \mathbf{A}\left(\nabla u_{H}\right) \cdot \mathbf{n} \rrbracket_{\gamma}\right|\right)+\left|S_{\gamma}\right| \varphi_{\left|\nabla u_{H}\left(T_{0}\right)\right|}\left(\left|s_{\gamma}\right|\right) \\
& \leq C_{\delta} C \int_{S_{\gamma}}\left|\mathbf{F}(\nabla u)-\mathbf{F}\left(\nabla u_{H}\right)\right|^{2} d x+C_{\delta} C \sum_{T \in N_{\gamma}} \operatorname{osc}^{2}\left(u_{H}, T\right) \\
& \quad+C_{\delta} C \sum_{T \in N_{\gamma}} \eta_{E}^{2}\left(u_{H}, T\right)+\delta C \sum_{T \in N_{\gamma}}\left|S_{\gamma}\right| \varphi_{\left|\nabla u_{H}(T)\right|}\left(\left|s_{\gamma}\right|\right) .
\end{aligned}
$$

For small $\delta>0$ the summand of the last term with $T=T_{0}$ could be absorbed on the left hand side, but the term with $T=T_{1}$ bothers us, since it has the wrong shift $\left|\nabla u_{H}\left(T_{1}\right)\right|$. With Corollary 26 and (3.27) we get rid of this term:

$$
\begin{aligned}
& \varphi_{\left|\nabla u_{H}\left(T_{1}\right)\right|}\left(\left|s_{\gamma}\right|\right) \leq C \varphi_{\left|\nabla u_{H}\left(T_{0}\right)\right|}\left(\left|s_{\gamma}\right|\right)+C\left|\llbracket \mathbf{F}\left(\nabla u_{H}\right) \rrbracket_{\gamma}\right|^{2} \\
& \leq C \varphi_{\left|\nabla u_{H}\left(T_{0}\right)\right|}\left(\left|s_{\gamma}\right|\right)+C\left(\varphi_{\left|\nabla u_{H}\left(T_{0}\right)\right|}\right)^{*}\left(\left|\llbracket \mathbf{A}\left(\nabla u_{H}\right) \cdot \mathbf{n} \rrbracket_{\gamma}\right|\right) .
\end{aligned}
$$

This and (3.28) gives for $\delta>0$ small

$$
\begin{aligned}
& \left|S_{\gamma}\right|\left(\varphi\left|\nabla u_{H}\left(T_{0}\right)\right|\right)^{*}\left(\left|\llbracket \mathbf{A}\left(\nabla u_{H}\right) \cdot \mathbf{n} \rrbracket_{\gamma}\right|\right) \\
& \quad \leq C\left\|\mathbf{F}(\nabla u)-\mathbf{F}\left(\nabla u_{H}\right)\right\|_{L^{2}\left(S_{\gamma}\right)}^{2}+C \sum_{T \in N_{\gamma}} \operatorname{osc}^{2}\left(u_{H}, T\right)+C \sum_{T \in N_{\gamma}} \eta_{E}^{2}\left(u_{H}, T\right) .
\end{aligned}
$$

Now, an application of (3.27) and $\left|S_{\gamma}\right| \sim h_{\gamma}|\gamma|$ prove the following assertion:

Lemma 10. For finite element solutions $u_{H}$ of (3.5) and $\gamma \in \Gamma_{H}$ it holds

$$
\eta_{J}^{2}\left(u_{H}, \gamma\right) \leq C\left\|\mathbf{F}\left(\nabla u_{H}\right)-\mathbf{F}(\nabla u)\right\|_{L^{2}\left(S_{\gamma}\right)}^{2}+C \operatorname{osc}^{2}\left(u_{H}, N_{\gamma}\right)+C \eta_{E}^{2}\left(u_{H}, N_{\gamma}\right),
$$

where the constant $C$ only depends on $\Delta_{2}(\varphi), \Delta_{2}\left(\varphi^{*}\right)$, and the shape regularity of $\mathcal{T}_{H}$.

Lemma 9 and Lemma 10 can be combined:

Corollary 11. For finite element solutions $u_{H}$ of (3.5) and $T \in \mathcal{T}_{H}$ it holds

$$
\eta^{2}\left(u_{H}, T\right) \leq C\left\|\mathbf{F}\left(\nabla u_{H}\right)-\mathbf{F}(\nabla u)\right\|_{L^{2}\left(S_{T}\right)}^{2}+C \operatorname{osc}^{2}\left(u_{H}, N_{T}\right),
$$

where the constant $C$ only depends on $\Delta_{2}(\varphi), \Delta_{2}\left(\varphi^{*}\right)$, and the shape regularity of $\mathcal{T}_{H}$. 
3.5. Discrete Lower Estimates. In the following let $\mathcal{T}_{h}$ be a refinement of $\mathcal{T}_{H}$, which is generated from $\mathcal{T}_{H}$ by finitely many bisections. Then $V_{h}:=V\left(\mathcal{T}_{h}\right)$ and $V_{H}:=V\left(\mathcal{T}_{H}\right)$ are nested, i.e. $V_{H} \subset V_{h} \subset V$. Let $u_{h}, u_{H}$ denote the unique solution of (2.1) with respect to $V_{h}$ respective $V_{H}$.

Our aim is to generalize Corollary 11 from $V_{H} \subset V$ to $V_{H} \subset V_{h}$. Therefore we have to ensure that $V_{h}$ is a sufficient refinement of $V_{H}$. In particular, we have to ensure the existence of bump functions as required in (3.13) and (3.22). We say that $T \in \mathcal{T}_{H}$ is fully refined in $\mathcal{T}_{h}$ if $T$ and each of its faces contains a node in its interior. This yields the existence of a bump function $w_{T} \in V_{h}$ on $T$ which satisfies (3.13) and bump functions $w_{\gamma} \in V_{h}$ on $S_{\gamma}$ for all $\gamma \in \Gamma_{H} \cup T$ which satisfy (3.22). Thus, to obtain the local lower bound (3.29) on $S_{T}$ for a certain $T \in \mathcal{T}_{H}$ it suffices to assume that each $T^{\prime} \in N_{T}$ is fully refined in $\mathcal{T}_{h}$. With these additional assumptions we can now transfer the estimates from Section 3.4 to $V_{H} \subset V_{h}$ :

Lemma 12. If for $T \in \mathcal{T}_{H}$ each $T^{\prime} \in N_{T}$ is fully refined in $\mathcal{T}_{h}$ then

$$
\eta^{2}\left(u_{H}, T\right) \leq C\left\|\mathbf{F}\left(\nabla u_{H}\right)-\mathbf{F}\left(\nabla u_{h}\right)\right\|_{L^{2}\left(S_{T}\right)}^{2}+C \operatorname{osc}^{2}\left(u_{H}, N_{T}\right),
$$

where the constant $C$ only depends on $\Delta_{2}(\varphi), \Delta_{2}\left(\varphi^{*}\right)$, and the shape regularity of $\mathcal{T}_{H}$.

\section{Algorithm And Convergence}

Let us first state the adaptive algorithm which produces a sequence $u_{k}$ of solutions in nested spaces $V_{k}:=V\left(\mathcal{T}_{k}\right)$ over triangulations $\mathcal{T}_{k}$. We substitute the index $H$, resp. $h$, of the previous sections by the Index $k$, resp. $k+1$, to indicate the underlying mesh. Then we introduce the concept of energy reduction and thereafter we prove convergence of the algorithm.

4.1. Adaptive Algorithm. We propose the following adaptive algorithm to solve (1.1):

Algorithm 13 (AFEM). Choose $\theta \in(0,1)$. Construct an initial triangulation $\mathcal{T}_{0}$ of $\Omega$ and set $k:=0$.

(1) ('Solve') Compute the solution $u_{k} \in V_{k}$ of Problem (3.5);

(2) ('Estimate') Compute $\eta^{2}\left(u_{k}, T\right)$ for all $T \in \mathcal{T}_{k}$.

(3) If $\eta^{2}\left(u_{k}, \mathcal{T}_{k}\right)=0$ then STOP;

(4) ('Mark') Choose a subset $\mathcal{T}_{k}^{m} \subset \mathcal{T}_{k}$ such that

$$
\eta^{2}\left(u_{k}, \mathcal{T}_{k}^{m}\right) \geq \theta^{2} \eta^{2}\left(u_{k}, \mathcal{T}_{k}\right) .
$$

(5) ('Refine') Perform a (minimal) conforming refinement of $\mathcal{T}_{k}$ using newest vertex bisections to obtain $\mathcal{T}_{k+1}$ such that each element of the neighbourhood $N\left(\mathcal{T}_{k}^{m}\right):=\bigcup_{T \in \mathcal{T}_{k}^{m}} N_{T} \subset \mathcal{T}_{k}$ of $\mathcal{T}_{k}^{m}$, is fully refined in $\mathcal{T}_{k+1}$. This ensures that each $T \in N\left(\mathcal{T}_{k}^{m}\right)$ as well as each of its faces contains a node of $\mathcal{T}_{k+1}$ in its interior. Increment $k$ and go to step (1).

Remark 14. Note that our marking strategy differs from the one proposed by Morin, Nochetto and Siebert in [MNS00]. They used separate marking steps for the error estimator and the data oscillation. In our setting this would correspond to the following strategy: Construct $\mathcal{T}_{k}^{m}$ as in step 'Mark'. Second, enlarge $\mathcal{T}_{k}^{m}$ such that for $\hat{\theta}>0$ also

$$
\operatorname{osc}^{2}\left(u_{k}, \mathcal{T}_{k}^{m}\right) \geq \hat{\theta}^{2} \operatorname{osc}^{2}\left(u_{k}, \mathcal{T}_{k}\right) .
$$

This requires the calculation of the oscillation in step 'Estimate'. We want to point out that by the marking strategy ('Mark') our new proof of convergence overcomes the drawback of additional marking for oscillation. This reflects the practical experience that the effect of oscillation plays a minor role (see e.g. [MNS00]). We 
prove the success of most adaptive strategies which disregard the issue of oscillation altogether. Since $\eta^{2}\left(u_{k}, T\right) \geq \operatorname{osc}^{2}\left(u_{k}, T\right)$, this implies that (4.1) is equivalent to

$$
\eta^{2}\left(u_{k}, \mathcal{T}_{k}^{m}\right)+\operatorname{osc}^{2}\left(u_{k}, \mathcal{T}_{k}^{m}\right) \geq \bar{\theta}^{2}\left(\eta^{2}\left(u_{k}, \mathcal{T}_{k}\right)+\operatorname{osc}^{2}\left(u_{k}, \mathcal{T}_{k}\right)\right)
$$

with $\bar{\theta} \in(0,1)$. Based on this cognitions we give a new proof to show that the combination of energy difference and oscillation is reduced in each step (see Theorem 20).

Remark 15. Note that the condition in 'Refine' of fully refined $T \in \mathcal{T}_{k}^{m}$ can be obtained by bisecting each $T \in \mathcal{T}_{k}^{m}$ three times in two dimensions respective six times in three dimensions (see [MNS00]). With this property we have a reduction factor $\lambda<1$ of element size, i.e. if $T^{\prime} \in \mathcal{T}_{k+1}$ is obtained by refining $T \in \mathcal{T}_{k}$ it holds $h_{T^{\prime}} \leq \lambda h_{T}$. By using the method of newest vertex bisection the shape regularity of $\left(\mathcal{T}_{k}\right)$ is uniformly bounded with respect to $k$ depending on the shape regularity of $\mathcal{T}_{0}$.

4.2. Energy Reduction in Nested Spaces. Assume as before that $V_{H} \subset V_{h} \subset$ $V$. One main ingredient of proving lineare convergence in [MNS00] for the linear case is the error reduction property for the energy norm

$$
\left\|u_{h}-u\right\|^{2}=\left\|u_{H}-u\right\|^{2}-\left\|u_{H}-u_{h}\right\|^{2} .
$$

This is a consequence of the Galerkin orthogonality and the Pythagorean Theorem which is related to Hilbert spaces. We do not have this property in the general case of the $\varphi$-Laplacian. But there is another way to interprete this property. In the linear case it is equivalent to

$$
\mathcal{J}\left(u_{h}\right)-\mathcal{J}(u)=\mathcal{J}\left(u_{H}\right)-\mathcal{J}(u)-\left(\mathcal{J}\left(u_{H}\right)-\mathcal{J}\left(u_{h}\right)\right) .
$$

Obviously, this equality holds also in our case. Since $V_{H} \subset V_{h} \subset V$ and the minimizing property of $u, u_{h}$, and $u_{H}$ we have

$$
\mathcal{J}(u) \leq \mathcal{J}\left(u_{h}\right) \leq \mathcal{J}\left(u_{H}\right) .
$$

Thus we have a reduction of energy difference. Now, it remains to find a link between the energy differences and the error. This is the content of the following Lemma. For $v, w \in V$ we define the energy difference by

$$
\varepsilon(v, w):=\mathcal{J}(v)-\mathcal{J}(w) .
$$

Lemma 16. Let $u_{1}, u_{2}$ be minimizers of the energy functional $\mathcal{J}$ with respect to the $V_{1} \subset V_{2} \subset V$. Then

$$
\mathcal{J}\left(u_{1}\right)-\mathcal{J}\left(u_{2}\right) \sim\left\|\mathbf{F}\left(\nabla u_{1}\right)-\mathbf{F}\left(\nabla u_{2}\right)\right\|_{2}^{2},
$$

where the constants only depend on $\Delta_{2}(\varphi), \Delta_{2}\left(\varphi^{*}\right)$, and (2.7).

Proof. Define $\Phi: \mathbb{R}^{d} \rightarrow \mathbb{R}$ by $\Phi(\mathbf{a}):=\varphi(|\mathbf{a}|)$ then $\mathcal{J}(u)=\int_{\Omega} \Phi(\nabla u) d x-\int_{\Omega} u f d x$. Let $g(t):=\mathcal{J}\left(\left[u_{2}, u_{1}\right]_{t}\right)$ for $t \in \mathbb{R}$, where $\left[u_{2}, u_{1}\right]:=(1-t) u_{2}+t u_{1}$. Since $u_{2}$ is the minimizer of $\mathcal{J}$ on $V_{2} \supset V_{1}$, we have $g^{\prime}(0)=0$. We estimate by Taylor's formula

$$
\begin{aligned}
& \mathcal{J}\left(u_{1}\right)-\mathcal{J}\left(u_{2}\right)=g(1)-g(0)=\frac{1}{2} \int_{0}^{1} g^{\prime \prime}(t)(1-t) d t \\
& \quad=\frac{1}{2} \sum_{k, m} \int_{0}^{1} \int_{\Omega}\left(\partial_{k} \partial_{m} \Phi\right)\left(\left[\nabla u_{2}, \nabla u_{1}\right]_{t}\right)\left(\partial_{k} u_{1}-\partial_{k} u_{2}\right)\left(\partial_{m} u_{1}-\partial_{m} u_{2}\right) d x(1-t) d t .
\end{aligned}
$$

Note that for $\mathbf{a}, \mathbf{b} \in \mathbb{R}^{d}$

$$
\sum_{k, m}\left(\partial_{k} \partial_{m} \Phi\right)(\mathbf{a}) b_{k} b_{m}=\frac{\varphi^{\prime}(|\mathbf{a}|)}{|\mathbf{a}|}\left(|\mathbf{b}|^{2}-\frac{|\mathbf{a} \cdot \mathbf{b}|}{|\mathbf{a}|^{2}}\right)+\varphi^{\prime \prime}(|\mathbf{a}|) \frac{|\mathbf{a} \cdot \mathbf{b}|^{2}}{|\mathbf{a}|^{2}}
$$


By Assumption 1 we have $c \varphi^{\prime}(t) \leq t \varphi^{\prime \prime}(t) \leq C \varphi^{\prime}(t)$ uniformly in $t \geq 0$. Therefor,

$$
\sum_{k, m}\left(\partial_{k} \partial_{m} \Phi\right)(\mathbf{a}) b_{k} b_{m} \leq(1+C) \frac{\varphi^{\prime}(|\mathbf{a}|)}{|\mathbf{a}|}|\mathbf{b}|^{2}
$$

and

$$
\sum_{k, m}\left(\partial_{k} \partial_{m} \Phi\right)(\mathbf{a}) b_{k} b_{m} \geq \frac{\varphi^{\prime}(|\mathbf{a}|)}{|\mathbf{a}|}|\mathbf{b}|^{2}+(c-1) \frac{\varphi^{\prime}(|\mathbf{a}|)}{|\mathbf{a}|} \frac{|\mathbf{a} \cdot \mathbf{b}|^{2}}{|\mathbf{a}|^{2}} \geq c \frac{\varphi^{\prime}(|\mathbf{a}|)}{|\mathbf{a}|}|\mathbf{b}|^{2} .
$$

In other words $\sum_{k, m}\left(\partial_{k} \partial_{m} \Phi\right)(\mathbf{a}) b_{k} b_{m} \sim \frac{\varphi^{\prime}(|\mathbf{a}|)}{|\mathbf{a}|}|\mathbf{b}|^{2}$ uniformly in $\mathbf{a}, \mathbf{b} \in \mathbb{R}^{d}$. Combining the last estimates with (4.5) yields

$$
\mathcal{J}\left(u_{1}\right)-\mathcal{J}\left(u_{2}\right) \sim \int_{0}^{1} \int_{\Omega} \frac{\varphi^{\prime}\left(\left|\left[\nabla u_{2}, \nabla u_{1}\right]_{t}\right|\right)}{\left|\left[\nabla u_{2}, \nabla u_{1}\right]_{t}\right|}\left|\nabla u_{1}-\nabla u_{2}\right|^{2} d x(1-t) d t .
$$

Now, we cite Lemma 19 from [DE05], which states that for any $\mathbf{a}, \mathbf{b} \in \mathbb{R}^{d}$

$$
\int_{0}^{1} \frac{\varphi^{\prime}\left(\left|[\mathbf{a}, \mathbf{b}]_{t}\right|\right)}{\left|[\mathbf{a}, \mathbf{b}]_{t}\right|} d t \sim \frac{\varphi^{\prime}(|\mathbf{a}|+|\mathbf{b}|)}{|\mathbf{a}|+|\mathbf{b}|}
$$

with constants only depending on $\Delta_{2}(\varphi)$ and $\Delta_{2}\left(\varphi^{*}\right)$. In particular, this, (4.6), Assumption 1, and Lemma 3 gives

$$
\begin{aligned}
\mathcal{J}\left(u_{1}\right)-\mathcal{J}\left(u_{2}\right) & \leq C \int_{\Omega} \frac{\varphi^{\prime}\left(\left|\nabla u_{2}\right|+\left|\nabla u_{1}\right|\right)}{\left|\nabla u_{2}\right|+\left|\nabla u_{1}\right|}\left|\nabla u_{1}-\nabla u_{2}\right|^{2} d x \\
& \leq C \int_{\Omega} \varphi^{\prime \prime}\left(\left|\nabla u_{2}\right|+\left|\nabla u_{1}\right|\right)\left|\nabla u_{1}-\nabla u_{2}\right|^{2} d x \\
& \leq C \int_{\Omega}\left|\mathbf{F}\left(\nabla u_{1}\right)-\mathbf{F}\left(\nabla u_{2}\right)\right|^{2} d x .
\end{aligned}
$$

On the other hand, (4.6), $\varphi^{\prime}(t) t \sim \varphi(t)$ by (2.6), and Jensen's inequality give

$$
\begin{aligned}
\mathcal{J}\left(u_{1}\right)-\mathcal{J}\left(u_{2}\right) & \geq c \int_{\Omega} \int_{0}^{1} \frac{\varphi\left(\left|\left[\nabla u_{2}, \nabla u_{1}\right]_{t}\right|\right)}{\left(\left|\nabla u_{2}\right|+\left|\nabla u_{1}\right|\right)^{2}}(1-t) d t\left|\nabla u_{1}-\nabla u_{2}\right|^{2} d x . \\
& \geq c \int_{\Omega} \frac{\varphi\left(\int_{0}^{1}\left|\left[\nabla u_{2}, \nabla u_{1}\right]_{t}\right| 2(1-t) d t\right)}{\left(\left|\nabla u_{2}\right|+\left|\nabla u_{1}\right|\right)^{2}}\left|\nabla u_{1}-\nabla u_{2}\right|^{2} d x .
\end{aligned}
$$

Uniformly in $\mathbf{a}, \mathbf{b} \in \mathbb{R}^{d}$ holds $\int_{0}^{1}\left|[\mathbf{a}, \mathbf{b}]_{t}\right| 2(1-t) d t \sim|\mathbf{a}|+|\mathbf{b}|$, because both sides are a norm for the couple $(\mathbf{a}, \mathbf{b})$. This and $\varphi^{\prime \prime}(t) t^{2} \sim \varphi(t)$ imply

$$
\mathcal{J}\left(u_{1}\right)-\mathcal{J}\left(u_{2}\right) \geq c \int_{\Omega} \varphi^{\prime \prime}\left(\left|\nabla u_{2}\right|+\left|\nabla u_{1}\right|\right)\left|\nabla u_{1}-\nabla u_{2}\right|^{2} d x .
$$

Now, Lemma 3 proves $\mathcal{J}\left(u_{1}\right)-\mathcal{J}\left(u_{2}\right) \geq c \int_{\Omega}\left|\mathbf{F}\left(\nabla u_{1}\right)-\mathbf{F}\left(\nabla u_{2}\right)\right|^{2} d x$.

We recall that by Algorithm 13 for each element $T \in \mathcal{T}_{k}^{m}$ it holds $N_{T} \subset N\left(\mathcal{T}_{k}^{m}\right)$. Thus each of elements in $N_{T}$ is marked for full refinement. Thus by Lemma 12 we have a discrete lower bound for each $T \in \mathcal{T}_{k}^{m}$. Summing over all sides in $\mathcal{T}_{k}^{m}$ yields together with Lemma 16:

Corollary 17. For the sequence of finite element solutions produced by Algorithm 13 holds

$$
\eta^{2}\left(u_{k}, \mathcal{T}_{k}^{m}\right) \leq C \varepsilon\left(u_{k}, u_{k+1}\right)+C \operatorname{osc}^{2}\left(u_{k}, N\left(\mathcal{T}_{k}^{m}\right)\right),
$$


where the constant $C$ only depends on $\Delta_{2}(\varphi), \Delta_{2}\left(\varphi^{*}\right),(2.7)$, and the shape regularity of $\mathcal{T}_{0}$.

4.3. Convergence. To prove that Algorithm 13 produces a sequence $\left(u_{k}\right)$, which converges to the weak solution $u$ of (1.1) we need an auxiliary lemma which deals with oscillation.

Lemma 18. Then for the sequence of finite element solutions produced by Algorithm 13 there exists $\rho \in(0,1)$ such that

$$
\operatorname{Osc}^{2}\left(u, \mathcal{T}_{k+1}\right) \leq \operatorname{osc}^{2}\left(u, \mathcal{T}_{k}\right)-\rho \operatorname{osc}^{2}\left(u, N\left(\mathcal{T}_{k}^{m}\right)\right),
$$

with $\rho=1-\lambda$ and $\lambda$ from Remark 15 .

Proof. Recall that for any $T \in \mathcal{T}_{k}$ and any $T^{\prime} \in \mathcal{T}_{k+1}$ with $T^{\prime} \subset T$ we have $h_{T^{\prime}} \leq h_{T}$. Moreover, if $T$ is refined in $\mathcal{T}_{k+1}$, then we even have $h_{T^{\prime}} \leq \lambda h_{T}$. In particular, this holds if $T \in \mathcal{T}_{k}^{m}$ since these $T$ are fully refined in $\mathcal{T}_{k+1}$. First for every $T \in \mathcal{T}_{k}$ we estimate

$$
\begin{aligned}
\sum_{T^{\prime} \in \mathcal{T}_{k+1}, T^{\prime} \subset T} \operatorname{osc}^{2}\left(u, T^{\prime}\right) & =\sum_{T^{\prime} \in \mathcal{T}_{k+1}, T^{\prime} \subset T} \inf _{f_{T^{\prime}} \in \mathbb{R}} \int_{T^{\prime}}(\varphi|\nabla u|)^{*}\left(h_{T^{\prime}}\left|f-f_{T^{\prime}}\right|\right) d x \\
& \leq \sum_{T^{\prime} \in \mathcal{T}_{k+1}, T^{\prime} \subset T} \inf _{f_{T^{\prime}} \in \mathbb{R}} \int_{T^{\prime}}\left(\varphi_{|\nabla u|}\right)^{*}\left(h_{T}\left|f-f_{T^{\prime}}\right|\right) d x \\
& \leq \inf _{f_{T} \in \mathbb{R}} \int_{T}(\varphi|\nabla u|)^{*}\left(h_{T}\left|f-f_{T}\right|\right) d x \\
& =\operatorname{osc}^{2}(u, T) .
\end{aligned}
$$

Second, for $T \in N\left(\mathcal{T}_{k}^{m}\right)$ we have a better estimate, since all elements in $N\left(\mathcal{T}_{k}^{m}\right)$ are fully refined in $\mathcal{T}_{k+1}$. Therefor, it holds with the convexity of $\mathrm{N}$-functions

$$
\begin{aligned}
\sum_{T^{\prime} \in \mathcal{T}_{k+1}, T^{\prime} \subset T} \operatorname{osc}^{2}\left(u, T^{\prime}\right) & =\sum_{T^{\prime} \in \mathcal{T}_{k+1}, T^{\prime} \subset T} \inf _{f_{T^{\prime}} \in \mathbb{R}} \int_{T^{\prime}}\left(\varphi_{|\nabla u|}\right)^{*}\left(h_{T^{\prime}}\left|f-f_{T^{\prime}}\right|\right) d x \\
& \leq \sum_{T^{\prime} \in \mathcal{T}_{k+1}, T^{\prime} \subset T} \inf _{f_{T^{\prime}} \in \mathbb{R}} \int_{T^{\prime}}\left(\varphi_{|\nabla u|}\right)^{*}\left(\lambda h_{T}\left|f-f_{T^{\prime}}\right|\right) d x \\
& \leq \inf _{f_{T} \in \mathbb{R}} \int_{T}(\varphi|\nabla u|)^{*}\left(\lambda h_{T}\left|f-f_{T}\right|\right) d x \\
& \leq \lambda \operatorname{osc}^{2}(u, T) .
\end{aligned}
$$

Now, (4.9) and (4.10) imply

$$
\begin{aligned}
\operatorname{osc}^{2}\left(u, \mathcal{T}_{k+1}\right) & \leq \operatorname{osc}^{2}\left(u, \mathcal{T}_{k} \backslash \mathcal{T}_{k}^{m}\right)+\lambda \operatorname{osc}^{2}\left(u, \mathcal{T}_{k}^{m}\right) \\
& =\operatorname{osc}^{2}\left(u, \mathcal{T}_{k}\right)-(1-\lambda) \operatorname{osc}^{2}\left(u, \mathcal{T}_{k}^{m}\right)
\end{aligned}
$$

This proves the Lemma.

Lemma 19. For the sequence of finite element solutions produced by Algorithm 13 holds

$$
\begin{aligned}
\eta^{2}\left(u_{k}, \mathcal{T}_{k}\right)+\operatorname{osc}^{2}\left(u_{k}, \mathcal{T}_{k}\right) & \sim \varepsilon\left(u_{k}, u\right)+\operatorname{osc}^{2}\left(u_{k}, \mathcal{T}_{k}\right) \\
& \sim \varepsilon\left(u_{k}, u\right)+\operatorname{osc}^{2}\left(u, \mathcal{T}_{k}\right) .
\end{aligned}
$$

Proof. First we prove (4.11a). From Corollary 11, Lemma 16, and Lemma 8 follows

$$
\begin{aligned}
\eta^{2}\left(u_{k}, \mathcal{T}_{k}\right) & \leq C \varepsilon\left(u_{k}, u\right)+C \operatorname{osc}^{2}\left(u_{k}, \mathcal{T}_{k}\right), \\
\varepsilon\left(u_{k}, u\right) & \leq C \eta^{2}\left(u_{k}, \mathcal{T}_{k}\right)+C \operatorname{osc}^{2}\left(u_{k}, \mathcal{T}_{k}\right),
\end{aligned}
$$


which immediately implies (4.11a). With the help of Corollary 28 we can change the shift in the last term, i.e.

$$
\begin{aligned}
& \operatorname{osc}^{2}\left(u_{k}, \mathcal{T}_{k}\right) \\
& \quad=\sum_{T \in \mathcal{T}_{k}} \inf _{f_{T} \in \mathbb{R}} \int_{T}\left(\varphi\left|\nabla u_{k}\right|\right)^{*}\left(h_{K}\left|f-f_{T}\right|\right) d x \\
& \leq \sum_{T \in \mathcal{T}_{k}}\left(\inf _{f_{T} \in \mathbb{R}} C \int_{T}\left(\varphi_{|\nabla u|}\right)^{*}\left(h_{K}\left|f-f_{T}\right|\right) d x+C\left|\mathbf{F}\left(\nabla u_{k}\right)-\mathbf{F}(\nabla u)\right|^{2}\right) \\
& =C \operatorname{osc}^{2}\left(u, \mathcal{T}_{k}\right)+C\left\|\mathbf{F}\left(\nabla u_{k}\right)-\mathbf{F}(\nabla u)\right\|_{2}^{2} \leq C \operatorname{osc}^{2}\left(u, \mathcal{T}_{k}\right)+C \varepsilon\left(u_{k}, u\right) .
\end{aligned}
$$

The same calculation with $u_{k}$ and $u$ exchanged proves

$$
\operatorname{osc}^{2}\left(u, \mathcal{T}_{k}\right) \leq C \operatorname{osc}^{2}\left(u_{k}, \mathcal{I}_{k}\right)+C \varepsilon_{k}\left(u_{k}, u\right) .
$$

Now, (4.12) and (4.13) proves (4.11b).

Now we are able to prove our main result.

Theorem 20 (Energy/Oscillation Reduction). There exist a constant $\alpha \in(0,1)$ such that for the sequence $\left(u_{k}\right)$ of finite elements solutions produced by Algorithm AFEM

$$
\varepsilon\left(u_{k+1}, u\right)+\operatorname{osc}^{2}\left(u, \mathcal{T}_{k+1}\right) \leq \alpha^{2}\left(\varepsilon\left(u_{k}, u\right)+\operatorname{osc}^{2}\left(u, \mathcal{T}_{k}\right)\right) .
$$

Here $\alpha$ only depends on the shape regularity of the sequence $\left(\mathcal{T}_{k}\right), \Delta_{2}(\varphi), \Delta_{2}\left(\varphi^{*}\right)$, (2.7), and $\theta$ from the marking strategy (4.1). In particular, for $k \in \mathbb{N}$

$$
\varepsilon\left(u_{k}, u\right)+\operatorname{osc}^{2}\left(u, \mathcal{T}_{k}\right) \leq \alpha^{2 k}\left(\varepsilon\left(u_{0}, u\right)+\operatorname{osc}^{2}\left(u, \mathcal{T}_{0}\right)\right) .
$$

Proof. We start with the energy reduction (4.4)

$$
\varepsilon\left(u_{k+1}, u\right)=\varepsilon\left(u_{k}, u\right)-\varepsilon\left(u_{k}, u_{k+1}\right)
$$

and note, that by Lemma 18 we have a similar relation for oscillation, i.e.,

$$
\operatorname{osc}^{2}\left(u, \mathcal{T}_{k+1}\right) \leq \operatorname{osc}^{2}\left(u, \mathcal{T}_{k}\right)-\rho \operatorname{osc}^{2}\left(u, N\left(\mathcal{T}_{k}^{m}\right)\right) .
$$

for some $\rho \in(0,1)$. Summing these two terms yields

$$
\begin{aligned}
& \varepsilon\left(u_{k+1}, u\right)+\operatorname{osc}^{2}\left(u, \mathcal{T}_{k+1}\right) \\
& \quad \leq \varepsilon\left(u_{k}, u\right)+\operatorname{osc}^{2}\left(u, \mathcal{T}_{k}\right)-\left(\varepsilon\left(u_{k}, u_{k+1}\right)+\rho \operatorname{osc}^{2}\left(u, N\left(\mathcal{T}_{k}^{m}\right)\right)\right) .
\end{aligned}
$$

The main idea is to apply the discrete lower bound to $\varepsilon\left(u_{k}, u_{k+1}\right)$, whereas the oscillation on the right hand side of (3.30) shall be absorbed at the oscillation term in the above inequality. Since in (3.30) the oscillation appears with shift $u_{k}$, we use Corollary 28 (change of shift) as in (4.12) to obtain

$$
\operatorname{osc}^{2}\left(u_{k}, N\left(\mathcal{T}_{k}^{m}\right)\right) \leq\left(1+C_{\delta}\right) C \operatorname{osc}^{2}\left(u, N\left(\mathcal{T}_{k}^{m}\right)\right)+\delta C \varepsilon_{k}\left(u_{k}, u\right)
$$

for $\delta>0$ and a corresponding $C_{\delta} \geq 0$. We set $D=\left(1+C_{\delta}\right)>1$ and obtain equivalently,

$$
\frac{1}{C D} \operatorname{osc}^{2}\left(u_{k}, N\left(\mathcal{T}_{k}^{m}\right)\right)-\frac{\delta}{D} \varepsilon\left(u_{k}, u\right) \leq \operatorname{osc}^{2}\left(u, N\left(\mathcal{T}_{k}^{m}\right)\right) .
$$

This, (4.17), and (4.7) imply

$$
\begin{aligned}
& \varepsilon\left(u_{k+1}, u\right)+\operatorname{osc}^{2}\left(u, \mathcal{T}_{k+1}\right) \\
& \quad \leq \varepsilon\left(u_{k}, u\right)+\operatorname{osc}^{2}\left(u, \mathcal{T}_{k}\right)-\left(\varepsilon\left(u_{k}, u_{k+1}\right)+\frac{\rho}{C D} \operatorname{osc}^{2}\left(u_{k}, N\left(\mathcal{T}_{k}^{m}\right)\right)-\frac{\rho \delta}{D} \varepsilon\left(u_{k}, u\right)\right) \\
& \leq\left(1+\frac{\rho \delta}{D}\right) \varepsilon\left(u_{k}, u\right)+\operatorname{osc}^{2}\left(u, \mathcal{T}_{k}\right)-\left(\varepsilon\left(u_{k}, u_{k+1}\right)+\frac{\rho}{C D} \operatorname{osc}^{2}\left(u_{k}, N\left(\mathcal{T}_{k}^{m}\right)\right)\right) .
\end{aligned}
$$


Since $D \geq 1$ and $\rho \in(0,1)$ we have $\frac{\rho}{2 C D} \leq 1$. Thus

$$
\begin{aligned}
& \varepsilon\left(u_{k+1}, u\right)+\operatorname{osc}^{2}\left(u, \mathcal{T}_{k+1}\right) \\
& \quad \leq\left(1+\frac{\rho \delta}{D}\right) \varepsilon\left(u_{k}, u\right)+\operatorname{osc}^{2}\left(u, \mathcal{T}_{k}\right)-\frac{\rho}{2 C D}\left(\varepsilon\left(u_{k}, u_{k+1}\right)+2 \operatorname{osc}^{2}\left(u_{k}, N\left(\mathcal{T}_{k}^{m}\right)\right)\right)
\end{aligned}
$$

the discrete lower estimate (4.7), and $\mathcal{T}_{k}^{m} \subset N\left(\mathcal{T}_{k}^{m}\right)$ yield

$$
\begin{aligned}
& \leq\left(1+\frac{\rho \delta}{D}\right) \varepsilon\left(u_{k}, u\right)+\operatorname{osc}^{2}\left(u, \mathcal{T}_{k}\right)-\frac{\rho}{2 C D}\left(\frac{1}{C} \eta^{2}\left(u_{k}, \mathcal{T}_{k}^{m}\right)+\operatorname{osc}^{2}\left(u_{k}, N\left(\mathcal{T}_{k}^{m}\right)\right)\right) \\
& \leq\left(1+\frac{\rho \delta}{D}\right) \varepsilon\left(u_{k}, u\right)+\operatorname{osc}^{2}\left(u, \mathcal{T}_{k}\right)-\frac{\rho}{2 C D}\left(\eta^{2}\left(u_{k}, \mathcal{T}_{k}^{m}\right)+\operatorname{osc}^{2}\left(u_{k}, \mathcal{T}_{k}^{m}\right)\right) .
\end{aligned}
$$

Now it remains to utilize the marking strategy. By the equivalent form (4.3) of our marking strategy 'Mark' and (4.11) we get

$$
C\left(\varepsilon\left(u_{k}, u\right)+\operatorname{osc}_{k}^{2}\left(u, \mathcal{T}_{k}\right)\right) \leq \bar{\theta}^{2}\left(\eta^{2}\left(u_{k}, \mathcal{T}_{k}^{m}\right)+\operatorname{osc}_{k}^{2}\left(u_{k}, \mathcal{T}_{k}^{m}\right)\right) .
$$

This observation and Lemma 8 finally imply

$$
\begin{aligned}
& \varepsilon\left(u_{k+1}, u\right)+\operatorname{osc}^{2}\left(u, \mathcal{T}_{k+1}\right) \\
& \leq\left(1+\frac{\rho \delta}{D}\right) \varepsilon\left(u_{k}, u\right)+\operatorname{osc}^{2}\left(u, \mathcal{T}_{k}\right)-\frac{\rho \bar{\theta}^{2}}{2 C D}\left(\eta^{2}\left(u_{k}, \mathcal{T}_{k}\right)+\operatorname{osc}^{2}\left(u, \mathcal{T}_{k}\right)\right) \\
& \leq\left(1+\frac{\rho \delta}{D}\right) \varepsilon\left(u_{k}, u\right)+\operatorname{osc}^{2}\left(u, \mathcal{T}_{k}\right)-\frac{\rho \bar{\theta}^{2}}{2 C D}\left(\frac{1}{C} \varepsilon\left(u_{k}, u\right)+\operatorname{osc}^{2}\left(u, \mathcal{T}_{k}\right)\right) \\
& \leq\left(1+\frac{\rho \delta}{D}-\frac{\rho \bar{\theta}^{2}}{2 C D}\right) \varepsilon\left(u_{k}, u\right)+\left(1-\frac{\rho \bar{\theta}^{2}}{2 C D}\right) \operatorname{osc}^{2}\left(u, \mathcal{T}_{k}\right) .
\end{aligned}
$$

Substituting $D$ by its definition, we set

$$
\alpha^{2}:=\min \left\{1+\frac{\rho \delta}{\left(1+C_{\delta}\right)}-\frac{\rho \bar{\theta}^{2}}{2 C\left(1+C_{\delta}\right)}, 1-\frac{\rho \bar{\theta}^{2}}{2 C\left(1+C_{\delta}\right)}\right\}
$$

and obtain the proposition for $\delta>0$ small enough.

From the energy/oscillation reduction of Theorem 20 it follows with the help of Lemma 16:

Corollary 21 (Energy/Oscillation Reduction). For the sequence $\left(u_{k}\right)$ of finite elements solutions produced by Algorithm AFEM there exists constant $\alpha \in(0,1)$ such that for all $k \in \mathbb{N}$

$$
\begin{aligned}
& \left\|\mathbf{F}\left(\nabla u_{k}\right)-\mathbf{F}(\nabla u)\right\|_{L^{2}(\Omega)}^{2}+\operatorname{osc}^{2}\left(u, \mathcal{T}_{k}\right) \\
& \quad \leq C \alpha^{2 k}\left(\left\|\mathbf{F}\left(\nabla u_{0}\right)-\mathbf{F}(\nabla u)\right\|_{L^{2}(\Omega)}^{2}+\operatorname{osc}^{2}\left(u, \mathcal{T}_{0}\right)\right) .
\end{aligned}
$$

Here $\alpha$ and the constants only depend on the shape regularity of $\mathcal{T}_{0}, \Delta_{2}(\varphi), \Delta_{2}\left(\varphi^{*}\right)$, (2.7), and $\theta$ from the marking strategy (4.1).

\section{Appendix}

In this section we summarize the properties of the shifted $\mathrm{N}$-functions $\varphi_{a}$. Recall that for given N-function $\varphi$ with $\Delta_{2}(\varphi), \Delta_{2}\left(\varphi^{*}\right)<\infty$ we define $\varphi_{a}$ as in (3.2) and $\mathbf{F}$ as in (3.1). The following results are from [DE05] and we present them here without proof. We use $C \geq 1$ as generic constant that does solely depend on the $\Delta_{2}$-constants of $\varphi$ respective $\varphi^{*}$ and may change from line to line. 
Lemma 22. Let $\varphi$ be an $N$-function with $\Delta_{2}(\varphi), \Delta_{2}\left(\varphi^{*}\right)<\infty$. Then for all $a \geq 0$ the functions $\varphi_{a}$ and $\left(\varphi_{a}\right)^{*}$ are $N$-function. Moreover, the families $\varphi_{a}$ and $\left(\varphi_{a}\right)^{*}$ satisfy the $\Delta_{2}$ condition uniformly in $\alpha \geq 0$, i.e. $c_{0}:=\sup _{a>0}\left(\Delta_{2}\left(\varphi_{a}\right), \Delta_{2}\left(\left(\varphi_{a}\right)^{*}\right)\right)<$ $\infty$. The constant $c_{0}$ depends on $\varphi$ only by $\Delta_{2}(\varphi)$ and $\Delta_{2}\left(\varphi^{*}\right)$. Moreover,

$$
\left(\varphi_{a}\right)^{*}(t) \sim\left(\varphi^{*}\right)_{\varphi^{\prime}(a)}(t)
$$

uniformly in $a, t \geq 0$, where the constants only depend on $\Delta_{2}(\varphi)$ and $\Delta_{2}\left(\varphi^{*}\right)$.

Lemma 23. Let $\varphi$ be a $N$-function with $\Delta_{2}(\varphi), \Delta_{2}\left(\varphi^{*}\right)<\infty$. Then uniformly in $\mathbf{a} \in \mathbb{R}^{d}$

$$
\begin{aligned}
\varphi^{\prime \prime}(|\mathbf{a}|+|\mathbf{b}|)|\mathbf{a}-\mathbf{b}| & \sim \varphi_{|\mathbf{a}|}^{\prime}(|\mathbf{a}-\mathbf{b}|) \sim \varphi_{|\mathbf{b}|}^{\prime}(|\mathbf{a}-\mathbf{b}|), \\
\varphi^{\prime \prime}(|\mathbf{a}|+|\mathbf{b}|)|\mathbf{a}-\mathbf{b}|^{2} & \sim \varphi_{|\mathbf{a}|}(|\mathbf{a}-\mathbf{b}|) \sim \varphi_{|\mathbf{b}|}(|\mathbf{a}-\mathbf{b}|),
\end{aligned}
$$

with constants only depending on $\Delta_{2}(\varphi)$ and $\Delta_{2}\left(\varphi^{*}\right)$.

Lemma 24. Let $\varphi$ be as in Assumption 1. Then $\varphi^{*}$ satisfies Assumption 1, too. If we define the $N$-function $\psi$ by

$$
\frac{\psi^{\prime}(t)}{t}:=\left(\frac{\varphi^{\prime}(t)}{t}\right)^{\frac{1}{2}}
$$

then $\psi$ and $\psi^{*}$ satisfy Assumption 1. Moreover, $\psi^{\prime \prime}(t) \sim \sqrt{\varphi^{\prime \prime}(t)}$ uniformly in $t>0$. The $\Delta_{2}$-constants of $\psi$ and $\psi^{*}$ and the constants of $\psi^{\prime \prime}(t) \sim \sqrt{\varphi^{\prime \prime}(t)}$ only depend on $\Delta_{2}(\varphi)$.

The following lemma is proven already in [DR06], but we give a shorter proof:

Lemma 25. Let $\varphi$ be a $N$-function with $\Delta_{2}(\varphi), \Delta_{2}\left(\varphi^{*}\right)<\infty$. Then there exists $C \geq 1$, which only depends on $\Delta_{2}(\varphi)$ such that for all $\mathbf{a}, \mathbf{b} \in \mathbb{R}^{d}$ and $t \geq 0$

$$
\varphi_{|\mathbf{a}|}^{\prime}(t) \leq C \varphi_{|\mathbf{b}|}^{\prime}(t)+C \varphi_{|\mathbf{a}|}^{\prime}(|\mathbf{a}-\mathbf{b}|)
$$

Proof. Since $\varphi_{|\mathbf{a}|}^{\prime}(t) \sim \varphi_{|\mathbf{a}|}(t) / t$ and $\varphi_{|\mathbf{a}|}(2 t) \sim \varphi_{|\mathbf{a}|}(t)$, we have $\varphi_{|\mathbf{a}|}^{\prime}(2 t) \sim \varphi_{|\mathbf{a}|}^{\prime}(t)$. In particular, $\varphi_{|\mathbf{a}|}^{\prime}(2 t) \leq C \varphi_{|\mathbf{a}|}^{\prime}(t)$ for some $C>0$ uniformly in $t \geq 0$. All constants depend only on $\Delta_{2}\left(\varphi_{|\mathbf{a}|}\right)$, so by Lemma 22 the constants depend only on $\Delta_{2}(\varphi)$.

Case $|\mathbf{a}-\mathbf{b}| \leq \frac{1}{2} t$ : From $|\mathbf{a}-\mathbf{b}| \leq \frac{1}{2} t$ follows $0 \leq \frac{1}{2}(|\mathbf{b}|+t) \leq|\mathbf{a}|+t \leq 2(|\mathbf{b}|+t)$. Hence,

$$
\varphi_{|\mathbf{a}|}^{\prime}(t)=\frac{\varphi^{\prime}(|\mathbf{a}|+t)}{|\mathbf{a}|+t} t \leq \frac{\varphi^{\prime}(2(|\mathbf{b}|+t))}{\frac{1}{2}(|\mathbf{b}|+t)} t \leq 2 C \frac{\varphi^{\prime}(|\mathbf{b}|+t)}{|\mathbf{b}|+t} t=2 C \varphi_{|\mathbf{b}|}^{\prime}(t) .
$$

Case $|\mathbf{a}-\mathbf{b}| \geq \frac{1}{2} t$ : We estimate

$$
\varphi_{|\mathbf{a}|}^{\prime}(t) \leq \varphi_{|\mathbf{a}|}^{\prime}(2|\mathbf{a}-\mathbf{b}|) \leq C \varphi_{|\mathbf{a}|}^{\prime}(|\mathbf{a}-\mathbf{b}|) .
$$

Combining the two cases proves the lemma.

Corollary 26 (Change of Shift). Let $\varphi$ be an $N$-function with $\Delta_{2}(\varphi), \Delta_{2}\left(\varphi^{*}\right)<\infty$. Then for any $\delta>0$ there exists $C_{\delta}>0$, which only depends on $\delta$ and $\Delta_{2}(\varphi)$ such that for all $\mathbf{a}, \mathbf{b} \in \mathbb{R}^{d}$ and $t \geq 0$

$$
\varphi_{|\mathbf{a}|}(t) \leq\left(1+C_{\delta}\right) C \varphi_{|\mathbf{b}|}(t)+\delta \varphi_{|\mathbf{a}|}(|\mathbf{a}-\mathbf{b}|) .
$$

If $\varphi$ satisfies Assumption 1, then for any $\delta>0$ there exists $C_{\delta}>0$, which only depends on $\delta, \Delta_{2}(\varphi), \Delta_{2}\left(\varphi^{*}\right)$, and (2.7) such that for all $\mathbf{a}, \mathbf{b} \in \mathbb{R}^{d}$ and $t \geq 0$

$$
\varphi_{|\mathbf{a}|}(t) \leq\left(1+C_{\delta}\right) C \varphi_{|\mathbf{b}|}(t)+\delta C|\mathbf{F}(\mathbf{a})-\mathbf{F}(\mathbf{b})|^{2} .
$$


Proof. Due to (2.7) holds $\varphi_{|\mathbf{a}|}(t) \sim \varphi_{|\mathbf{a}|}^{\prime}(t) t$. Now inequality (5.4) follows by (5.3), an application of Young's inequality (2.3), (5.2), and (2.5)

$$
\begin{aligned}
\varphi_{|\mathbf{a}|}(t) & \leq C \varphi_{|\mathbf{a}|}^{\prime}(t) t \leq C \varphi_{|\mathbf{b}|}^{\prime}(t) t+C \varphi_{|\mathbf{a}|}^{\prime}(|\mathbf{a}-\mathbf{b}|) t \\
& \leq C \varphi_{|\mathbf{b}|}(t)+C \delta \varphi_{|b|}^{*}\left(\varphi_{|\mathbf{a}|}^{\prime}(|\mathbf{a}-\mathbf{b}|)\right)+C C_{\delta} \varphi_{|\mathbf{b}|}(t) .
\end{aligned}
$$

On the other hand (5.5) follows from (5.4) with the help of Lemma 3.

The following lemma is new. It generalizes the change of shift to complementary functions.

Lemma 27. If $\varphi$ satisfies Assumption 1, then for any $\delta>0$ there exists $C_{\delta}>0$, which only depends on $\delta, \Delta_{2}(\varphi)$, and $\Delta_{2}\left(\varphi^{*}\right)$, such that for all $\mathbf{a}, \mathbf{b} \in \mathbb{R}^{d}$ and $t \geq 0$

$$
\left(\left(\varphi_{|\mathbf{a}|}\right)^{*}\right)^{\prime}(t) \leq C\left(\left(\varphi_{|\mathbf{b}|}\right)^{*}\right)^{\prime}(t)+C|\mathbf{a}-\mathbf{b}| \text {. }
$$

Proof. With Lemma 22, $|\mathbf{A}(\mathbf{a})|=\varphi^{\prime}(|\mathbf{a}|)$, Lemma 25, Lemma 3, and $\Delta_{2}(\varphi)<\infty$ we estimate

$$
\begin{aligned}
\left(\left(\varphi_{|\mathbf{a}|}\right)^{*}\right)^{\prime}(t) & \leq C\left(\left(\varphi^{*}\right)_{|\mathbf{A}(\mathbf{a})|}\right)^{\prime}(t) \\
& \leq C\left(\left(\varphi^{*}\right)_{|\mathbf{A}(\mathbf{a})|}\right)^{\prime}(t)+C\left(\left(\varphi^{*}\right)_{|\mathbf{A}(\mathbf{a})|}\right)^{\prime}(|\mathbf{A}(\mathbf{a})-\mathbf{A}(\mathbf{b})|) \\
& \leq C\left(\left(\varphi_{|\mathbf{a}|}\right)^{*}\right)^{\prime}(t)+C\left(\left(\varphi_{|\mathbf{a}|}\right)^{*}\right)^{\prime}\left(C \varphi_{|\mathbf{a}|}^{\prime}(|\mathbf{a}-\mathbf{b}|)\right) \\
& \leq C\left(\left(\varphi_{|\mathbf{a}|}\right)^{*}\right)^{\prime}(t)+C\left(\left(\varphi_{|\mathbf{a}|}\right)^{*}\right)^{\prime}\left(\varphi_{|\mathbf{a}|}^{\prime}(|\mathbf{a}-\mathbf{b}|)\right) \\
& =C\left(\left(\varphi_{|\mathbf{a}|}\right)^{*}\right)^{\prime}(t)+C|\mathbf{a}-\mathbf{b}| .
\end{aligned}
$$

This proves the Lemma.

Corollary 28 (Change of Shift $*$ ). If $\varphi$ satisfies Assumption 1, then for any $\delta>0$ there exists $C_{\delta}>0$, which only depends on $\delta, \Delta_{2}(\varphi)$, and $\Delta_{2}\left(\varphi^{*}\right)$, such that for all $\mathbf{a}, \mathbf{b} \in \mathbb{R}^{d}$ and $t \geq 0$

$$
\left(\varphi_{|\mathbf{a}|}\right)^{*}(t) \leq\left(1+C_{\delta}\right) C\left(\varphi_{|\mathbf{b}|}\right)^{*}(t)+\delta C \varphi_{|\mathbf{a}|}(|\mathbf{a}-\mathbf{b}|) .
$$

If $\varphi$ satisfies Assumption 1, then for any $\delta>0$ there exists $C_{\delta}>0$, which only depends on $\delta, \Delta_{2}(\varphi), \Delta_{2}\left(\varphi^{*}\right)$, and (2.7) such that for all $\mathbf{a}, \mathbf{b} \in \mathbb{R}^{d}$ and $t \geq 0$

$$
\left(\varphi_{|\mathbf{a}|}\right)^{*}(t) \leq\left(1+C_{\delta}\right) C\left(\varphi_{|\mathbf{b}|}\right)^{*}(t)+\delta C|\mathbf{F}(\mathbf{a})-\mathbf{F}(\mathbf{b})|^{2} .
$$

Proof. Due to $(2.7)$ holds $\left(\varphi_{|a|}\right)^{*}(t) \sim\left(\left(\varphi_{|a|}\right)^{*}\right)^{\prime}(t) t$. Now, inequality (5.7) follows by (5.6), an application of Young's inequality (2.3) (5.2), and (2.5) similarly to the proof of Corollary 26. On the other hand (5.8) follows from (5.7) with the help of Lemma 3.

\section{REFERENCES}

[BL93a] J. W. Barrett and W. B. Liu, Finite element approximation of the p-Laplacian, Math. Comp. 61 (1993), no. 204, 523-537.

[BL94a] J. W. Barrett and W. B. Liu, Finite element approximation of degenerate quasilinear elliptic and parabolic problems, Numerical analysis 1993 (Dundee, 1993), Pitman Res. Notes Math. Ser., vol. 303, Longman Sci. Tech., Harlow, 1994, pp. 1-16.

[BL94b] J.W. Barrett and W.B. Liu, Finite element approximation of the parabolic p-Laplacian, Siam J. Numer. Anal. 31 (1994), 413-428.

[CLY06] C. Carstensen and W. B. Liu and N. Yan, A posteriori FE error control for p-Laplacian by gradient recovery in quasi-norm, Math. Comput. 75 (1994), no. 256, 1599-1616.

[CK06] C. Carstensen and R. Klose, A posteriori finite element error control for the p-Laplace problem, SIAM J. Numer. Anal. 25 (2003), no. 3, 792-814.

[DE05] L. Diening and F. Ettwein, Fractional estimates for non-differentiable elliptic systems with general growth, Forum Mathematicum, accepted (2006).

[DR06] L. Diening and M. Růžička, Error estimates for interpolation operators in Orlicz-Sobolev spaces and quasi norms, Preprint, Mathematische Fakultät, Albert-Ludwigs-Universität Freiburg (2006). 
[Dör96] W. Dörfler, A convergent adaptive algorithm for Poisson's equation, SIAM J. Numer. Anal. 33 (1996), no. 3, 1106-1124.

[Ebm05] C. Ebmeyer, Gobal regularity in Sobolev spaces for elliptic problems with p-structure on bounded domains, Trends in partial differential equations of mathematical physics, Progr. Nonlinear Differential Equations Appl., vol. 61, Birkhäuser, Basel, 2005, pp. 81-89.

[EL05] C. Ebmeyer and WB. Liu, Quasi-norm interpolation error estimates for the piecewise linear finite element approximation of p-Laplacian problems, Numer. Math. 100 (2005), no. 2, 233-258.

[ELS05] C. Ebmeyer, W. B. Liu, and M. Steinhauer, Global regularity in fractional order Sobolev spaces for the p-Laplace equation on polyhedral domains, Z. Anal. Anwendungen 24 (2005), no. 2, 353-374.

[Giu03] E. Giusti, Direct methods in the calculus of variations, World Scientific Publishing Co. Inc., River Edge, NJ, 2003.

[GM75] R. Glowinski and A. Marocco, Sur l'approximation par éléments finis d'ordre un, et la résolution, par pénalisation-dualité, d'une classe de problèmes de Dirichlet non lineaires, Rev. Française Automat. Informat. Recherche Opérationelle Ser. Rouge Anal. Numér. 2 (1975), 41-76.

[LY01] W. Liu and N. Yan, Quasi-norm local error estimators for p-Laplacian, SIAM J. Numer. Anal. 39 (2001), no. 1, 100-127 (electronic).

[LY02] On quasi-norm interpolation error estimation and a posteriori error estimates for p-Laplacian, SIAM J. Numer. Anal. 40 (2002), no. 5, 1870-1895 (electronic).

[MN05] K. Mekchay and R. H. Nochetto, Convergence of adaptive finite element methods for general second order linear elliptic PDEs, SIAM J. Numer. Anal. 43 (2005), no. 5, 1803-1827 (electronic).

[MNS00] P. Morin, R. Nochetto, and K. Siebert, Data oscillation and convergence of adaptive FEM, Siam J. Numer. Anal. 38 (2000), 466-488.

[RR91] M. M. Rao and Z. D. Ren, Theory of Orlicz spaces, Monographs and Textbooks in Pure and Applied Mathematics, vol. 146, Marcel Dekker Inc., New York, 1991.

[SZ90] L. Ridgway Scott and S. Zhang, Finite element interpolation of nonsmooth functions satisfying boundary conditions, Math. Comp. 54 (1990), no. 190, 483-493.

[Ve02] A.Veeser, Convergent adaptive finite elements for the nonlinear Laplacian, Numer. Math. 92 (2002), no. 4, 743-770.

Lars Diening, Abteilung für angewandte Mathematik, Eckerstr. 1, 79104 Freiburg i. Br., Germany.

URL: http://www.mathematik.uni-freiburg.de/IAM/homepages/diening

E-mail address: diening@mathematik.uni-freiburg.de

Christian Kreuzer, , Institut für Mathematik, Universitätsrasse 14, 86159 Augsburg, Germany.

URL: http://scicomp.math.uni-augsburg.de/ kreuzer/

E-mail address: kreuzer@math.uni-augsburg.de 